\title{
Time-Resolved Production and Detection of Reactive Atoms
}

\author{
L. W. Grossman \\ G. S. Hurst
}




\section{DISCLAIMER}

This report was prepared as an account of work sponsored by an agency of the United States Government. Neither the United States Government nor any agency Thereof, nor any of their employees, makes any warranty, express or implied, or assumes any legal liability or responsibility for the accuracy, completeness, or usefulness of any information, apparatus, product, or process disclosed, or represents that its use would not infringe privately owned rights. Reference herein to any specific commercial product, process, or service by trade name, trademark, manufacturer, or otherwise does not necessarily constitute or imply its endorsement, recommendation, or favoring by the United States Government or any agency thereof. The views and opinions of authors expressed herein do not necessarily state or reflect those of the United States Government or any agency thereof. 


\section{DISCLAIMER}

Portions of this document may be illegible in electronic image products. Images are produced from the best available original document. 


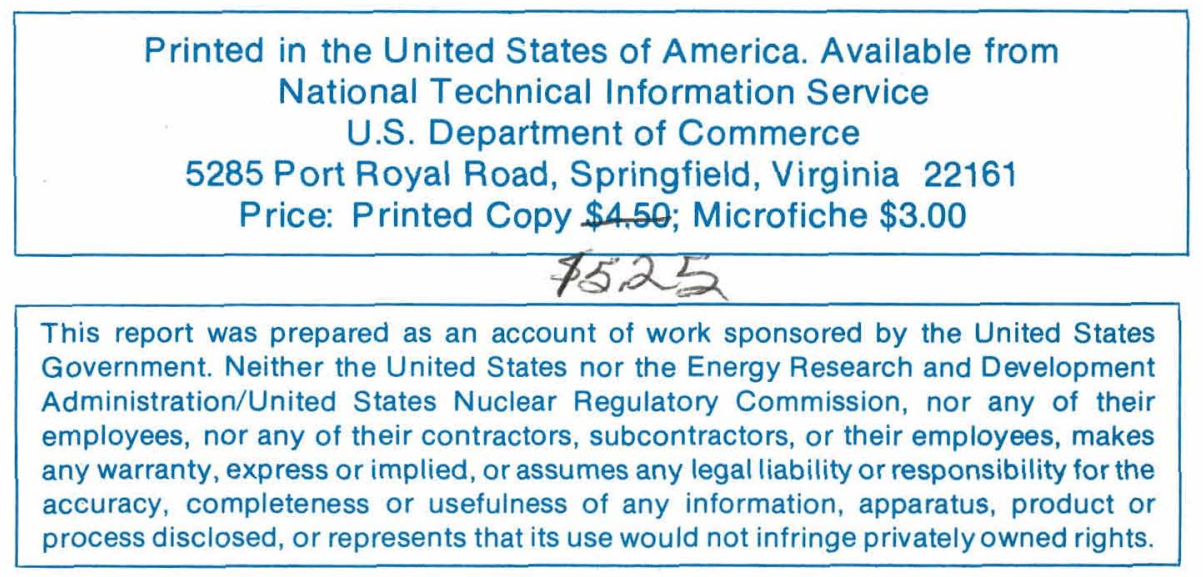


ORNL/TM-6014

Contract No. W-7405-eng-26

HEALTH AND SAFETY RESEARCH DIVISION

TIME-RESOLVED PRODUCTION AND DETECTION OF REACTIVE ATOMS

L. W. Grossman and G. S. Hurst

Submitted by L. W. Grossman as a dissertation to the Graduate School of the University of Kentucky in partial fulfillment of the requirements

for the degree of

Doctor of Philosophy

Date Published - September 1977

OAK RIDGE NATIONAL LABORATORY

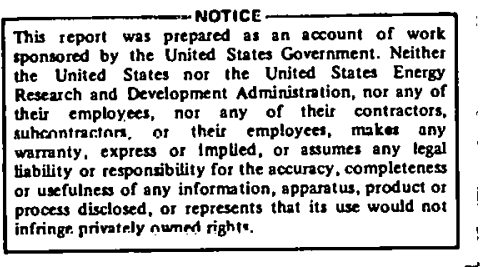

Oak Ridge, Tennessee 37830

operated by

UNION CARBIDE CORPORATION

for the

ENERGY RESEARCH AND DEVELOPMENT ADMINISTRATION 


\section{THIS PAGE}

\section{WAS INTENTIONALLY \\ LEFT BLANK}


TABLE OF CONTENTS

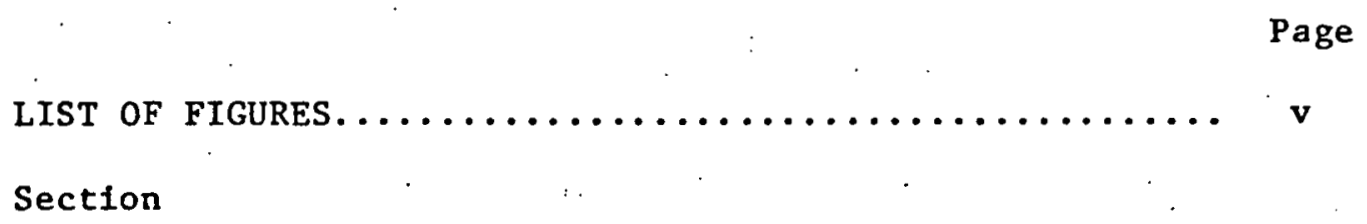

1. INTRODUCTION........................ 1

2. EXPERIMENTAL METHOD

2.1 Concept of the Experiment............ 6

2.2 Electronics and Data Acquistion......... 8

2.3 The Interaction Cell............... 11

2.4 The Lasers..................... 13

3. EXPERIMENTAL RESULTS AND DISCUSSTON

3.1. Photoionization of Cesium............ 17

3.2 Saturated Photodissociation............ 26

3.3 Diffusion of Cesium in Argon........... 32

3.4 The Reaction of Free Cesium with Oxygen in an Argon Atmosphere................ 39

4. SUMMARY......................... 46

5. REFERENCES........................ 49 


\section{THIS PAGE}

\section{WAS INTENTIONALLY \\ LEFT BLANK}


1. The Interaction Cell.................... 7

2. The Data Acquisition Hardware............... 9

3. Relative Intensity Vs. Wavelength of a Single Pulse of the Detector Laser.................... 14

4. Spectrum Wavelengths, Transition Rates and Lifetimes for Lower Levels of the Cesium Atom........ 19

5. Voltage Saturation Curve of Cesium in Argon....... 21

6. Ionization Signa1. Vs. Detector Laser Intensity Using Fresh Dye...................... 23

7. Ionization Signal Vs. Detector Laser Intensity Using Aged Dye........................ 24

8. Ionization Signal Vs. Photons Per Pulse of the Source Laser......................... 29

9. Cross Section for the Photoproduction of Cesium as a Function of Wavelength.................. 31

10. Relative Cesium Atom Signal as a Function of Time in 25 Torr of Pure Argon.................. 35

11. Relative Cesium Atom Signal as a Function of Time in 50 Torr of Pure Argon................ 37

12. Relative Cesium Atom Signal as a Function óf Time For Various Partial Pressures of Oxygen in

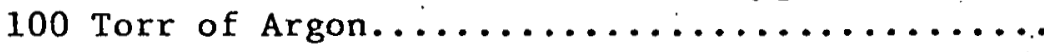




\section{THIS PAGE \\ WAS INTENTIONALLY \\ LEFT BLANK}




\section{ACKNOWLEDGEMENTS}

The authors express much appreciation to M. G. Payne for his theoretical guidance; to C. H. Chen, J. P. Judish, S. D. Kramer, M. H. Nayfeh, E. B. Wagner, and J. P. Young for their help with the experiments; and especially to S. L. Allman for assembly of the ionization chamber as well as continued assistance throughout the investigation. 


\section{THIS PAGE}

\section{WAS INTENTIONALLY LEFT BLANK}




\section{ABSTRACT}

A series of experiments were performed in which a population of free cesium atoms was created by photodissociation and then detected by photoionization. Two pulsed lasers in a coaxial geometry were employed. The first laser photodissociated cesium iodide molecules with a pulse of ultraviolet photons and is called the source laser. The second laser, using a technique known as resonance ionization spectroscopy, photoionized the cesium atoms within its beam and is called the detector laser. The laser beams passed through the center of a simple parallel plate ionization gas cell filled with argon. The ionization that occurred within a cylinder defined by the laser beam and the guarded ionization plate was collected and measured for each pulse.

Study of the ionization yield as a function of detector laser intensity indicates the process can be strongly saturated; that is, all of the cesium atoms within the ionization cylinder can be ionized. Study of the dissociation process with a focused and thus extremely intense source pulse indicates that in the central portion of the beam this process is also saturated. These results, combined with a previously demonstrated ability to detect single atoms of cesium, prove the ability to detect single molecules of cesium iodide.

Saturation of the dissociation process provides a measure of the cesium iodide concentration. By studying relative ionization as a function of photons per pulse of the source laser, and by accounting for the laser beam intensity profile, it was possible to determine the absolute cross section for photodissociation of cesium iodide into its 
ground-state atomic constituents. Knowledge of the vapor pressure of cesium iodide was not required, thus obviating the task of constructing an isothermal saturated vapor pressure cell. Absolute cross sections for photodissociation of CsI as a function of wavelength are reported. The dissociation/ionization technique has an excellent signal-tonoise ratio even in a simple parallel-plate ionization chamber. By saturating the ionization, signal dependence on buffer gas pressure or detector laser intensity is eliminated. Furthermore, the 1onfzatiun pulse amplitude is linearly related to the absolute number of cesium atoms in the ionization beam. Therefore, the decay in number of free cesium atoms within the ionization cylinder can be followed for many orders of magnitude.

Using a tightly focused source laser, free ceslum atums wert created along the axis of the ionization cylinder. The functional form of diffusion transport out of the cylinder, accounting also for reactions with impurities, is easily calculated. Diffusion transport was found to dominate the decay of cesium atoms out of the lonization cylinder at argon pressures below 100 torr. The extraction of a diffusion coefficient was then accomplished rather directly by data fitting. The diffusion coefficient of cesium in argon was found to be $0.12 \pm 0.03$ $\mathrm{cm}^{2}-\mathrm{sec}^{-1}$ at atmospheric pressure. More conventiunal Lechuiques for the determination of diffusion coefficients require the gaseous components to be inert and noncondensing at the container walls. However, in the present study this requirement was relaxed because diffusion was observed on a millisecond time scale far from the walls of the counter. 
The reaction of cesium with oxygen was observed by adding small partial pressures of oxygen to the argon buffer gas. Oxygen pressures were selected so that the resulting reactive decay occurred on a microsecond time scale. The diffusion processes had a negligible influence on the time dependence of the cesium decay. A three-body reaction rate of $(7.5 \pm 0.7) \times 10^{-30} \mathrm{sec}^{-1}$-particle ${ }^{-2}-\mathrm{cm}^{6}$ was determined. This rate is appropriate to argon pressures as high as 100 torr. The ionization cylinder temperature was $325 \mathrm{~K}$. 


\section{SECTION 1}

\section{INTRODUCTION}

Resonance ionization spectroscopy (RIS) is a highly sensitive technique for the measurement of absolute populations of atomic species. In its simplest form, RIS is accomplished by exciting atoms of the desired state to an intermediate state by absorption of a photon from a laser tuned to this transition wavelength. Absorption of an additional photon from the same laser can promote the intermediate. state to the ionization continuum. If a high intensity pulsed laser is used, all of the atoms of the desired state can be photoionized. The rate limiting step in this case is the ionization probability of the excited state.

The first use of RIS was a measurement of the absolute population of metastable $\mathrm{He}\left(2^{1} \mathrm{~S}\right)$ following excitation with a proton pulse ${ }^{1}$. Each $\mathrm{He}\left(2^{l} S\right)$ was excited to $\mathrm{He}\left(3^{l} \mathrm{P}\right)$ and then ionized by a pulsed laser tuned to $5015 \AA$. To obtain photoionization saturation, a photon fluence of $2.5 \times 10^{18}$ photons per $\mathrm{cm}^{2}$ per pulse was necessary. The actual ionization kinetics can be a great deal more complicated than described so far. In the case of $\mathrm{He}\left(2^{1} \mathrm{~S}\right)$, study of the ionization yield as a function of pressure ${ }^{2}$ indicated that at pressures of greater than 15 torr, collision-induced associative ionization of the He( $\left.3^{l} \mathrm{P}\right)$ state occurred and saturation of ionization could be produced at lower power leve1s. 
Recent work demonstrated the ability to detect single atoms of cesium with the RIS technique ${ }^{3,4}$. In this experiment, cesium atoms were volatilized from a source and allowed to diffuse into a proportional counter filled with P10 gas ( $90 \%$ argon and $10 \%$ methane). The counter was coupled to low noise electronics, providing a system sensitive to a single free electron. A pulsed laser tuned to $4555 \AA^{\circ}$ was used to excite to the $7^{2} \mathrm{P}_{3 / 2}$ level and then ionize all the cesium within a beam volume of $5 \times 10^{-2} \mathrm{~cm}^{3}$. 'l'hè cestum sulle strength wao reduced until one cesium fonization event was counted per twenty laser pulses, indicating the average concentration to be inuch less than one atom. This experiment also demonstrated the high selectivity of the RIS process when it is considered the ionization volume contained $10^{17}$ atoms and molecules of counting gas.

Because of energy level spacingo, most eloments rannnt he readily ionized by a simple single-laser two-photon process in spite of the availability of pulsed lasers with tunable outputs in the ultraviolet, visible, and infrared spectral regions 5 . However, multiple lasers can be used to promote two or more resonant steps. In addition, recent work indicates that multi-photon resonant transitions followed by ionization can be saturaced will currently avallabl \& laser systems ${ }^{6}$. Therefore, RIS techniques could be applied to a sizable fraction of the periodic table.

During the course of the cesium RIS work it became desirable to gain some knowledge of the transport and decay of cesium vapor in a typical gas atmosphere. Systematic studies were not found of cesium 
reactivity appropriate to the conditions encountered in the oneatom experiments. Therefore, this work was undertaken to develop a simple method to determine reaction rates of cesium vapor with typical counting gas impurities such as oxygen. Since the RIS technique provides an excellent method for monitoring the atomic cesium population, all that was required was a means of introducing cesium into a known amount of impurity within the RIS 1aser beam.

Absorption of ultraviolet light has long been known to cause dissociation of a diatomic alkali-halide molecule into its atomic constituents 7,8 . The dominant feature of alkali-halide absorption is a series of broad continua. Starting on the long wavelength side, the first continuum is interpreted as resulting in excitation to an intermediate molecular excited state which dissociates into ground state atoms. Absorption continua further into the ultraviolet are due to dissociation into particular excited states of either the alkali or halide atoms. Studies of the shape of the absorption continua resulted in estimates of the excited molecular potentials $9,10,11$.

The technique of using photodissociation to create a population of atoms in a reactive atmosphere was first used by Terenin ${ }^{12}$. He studied the reaction of sodium with iodine vapor. More recently, the same technique has been used to study several alkali metal reactions with halogens 13,14 .

In this study, cesium iodide in the presence of a buffer gas was dissociated with a pulsed ultraviolet laser, which will be referred to as the source laser. This created a population of atoms at a well: 
defined time and in a compact, well defined, volume. A second pulsed laser, with a beam.that completely surrounded that of the first, photoionized the cesium after a known time delay. This laser will be referred to as the detector 1aser. It was determined that. for short time delays, all of the cesium atoms were easily ionized. When focused, the source laser generated an extremely intense fluence. By accounting for the beam intensity profile it was shown that all of the molecules in the central portion of the beam can be dissociated and detected. Besides proving the feasibility of single-molecule detection, this enabled a determination of the absolute phuludissociation cross section as a function of wavelength. No independent knowledge of the number density of cesium iodide molecules was required.

Gas diffusion studies have been the subject of considerable. interest in the past ${ }^{15}$ and are also of current interest ${ }^{16}$. Conventional techniques for measuring diffusion coefficients require the gas constituents to be inert and non-condensing at the walls of the container vessel. For māny materlals llese conditions can only be achieved at high temperatures and are difficult even then: Condensable systems require special approaches so that diffusion can be studied far from container walls. Because of this, there are few measureuleuts of alkali metal diffusion in gases.

Initial studies of the time decay of the cesium signal at low argon pressures indicated a non-exponential decay. This was consistent with a diffusion mechanism transporting cesium atoms out of the laser. beam. Therefore, it was desired to conduct further experiments using 
a tightly focused source beam, passing along the axis of the detector beam. The theoretical behavior of this simple geometry accounting for diffusion and reaction is easily calculated. A diffusion coefficient can then be extracted by data fitting. If reactive decay is due to impurities constituting a fixed percentage of the buffer gas, then twobody reaction rates will scale linearly with pressure and three-body reaction rates will scale quadratically. Also, the diffusion coefficient will scale inversely with pressure. At low pressures it is conceivable that decay due to diffusion would be sufficiently rapid that all other processes can be neglected. Extraction of a diffusion coefficient would then be quite direct.

Finally, study of the reaction of cesium and oxygen was undertaken. Small partial pressures of oxygen were added to the argon buffer gas. The resulting time decays were sufficiently fast that diffuston losses were completely negligible. A three-body reaction rate for this system was obtained. 
SECTION 2

EXPERIMENTAL METHOD

\subsection{A Concept of the Experiment}

The experimental arrangement (Figure 1) utilizes a parallel plate ionization chamber mounted inside an inert gas cell. Cesium iodide vapor is generated by heating a sample cup plated with CsI crystals beneath the ionization plates and allowing the vapor to diffuse through the buffer gas into the active volume. A narrow pulsed beam of UV light obtained from a frequency-doubled tunable dye laser is passed through the center of the ionization region. This UV pulse generates neutral atomic cesium via the photodissociation process and is referred to in the rext. as the source beam. After a kuuwn time delay, a oooond laser beam of much larger diameter is pulsed through the cell along the same axis as the first. This second laser is tuned to ionize Cs to saturation, and is called the detector beam. The active volume of the cell is a cylinder defined by the area of the detector beam and the length of the guarded ionization plate. The electrons produced by the ionization are collected at the guarded plate. This pulse of charge is amplified and stored for readout by the electronlcs. Thus, at time $t=0$, free cs atoms are produced along the axis of a cylinder. At some later time, $\dot{t}=\tau$, the ionization laser detects those atoms that have 


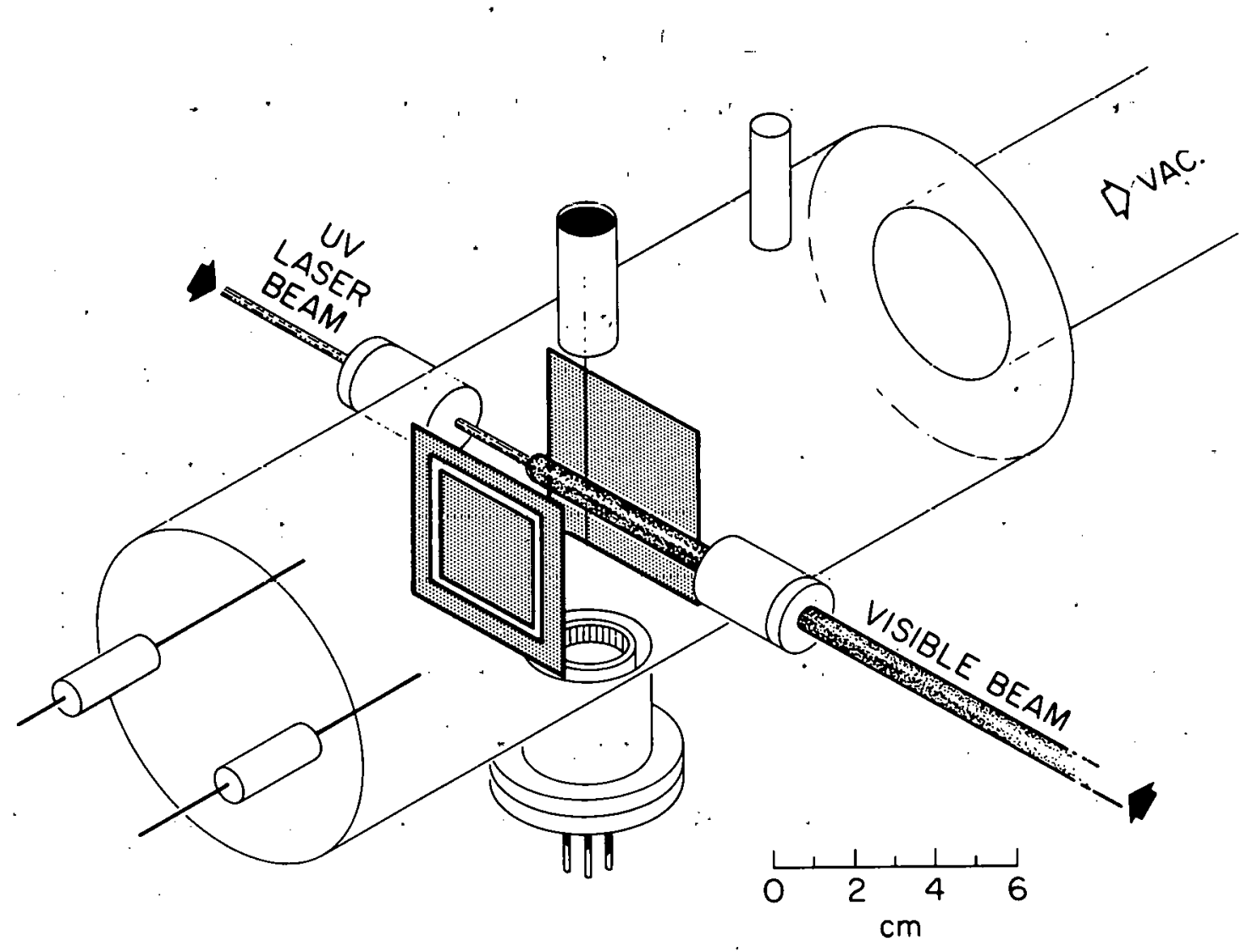

Flgure 1. The interaction cell. The UV beam photodissociates cesium iodide. The visible beam ionizes free cesium. 
not diffused out of the cylinder or reacted with impurities in the inert buffer gas. Data is generated by repeating the sequence many times while varying a single parameter, such as time delay or laser intensity.

\subsection{Electronics and Data Acquisition}

The experimental electronics control the dissociation-toionization time delay and, in addition, store three pieces of information for each pulse sequence (FIgure 2). The three pieces of information are: (1) a signal proportional to the energy per pulse of the source laser; (2) a signal proportional to the energy per pulse of the detector laser; and, (3) the resonance ionization signal. Just before the pulsed source beam enters the reaction cell, a small fraction is picked off with a quartz beam splitter and directed onto a filuorescent card. A light sensitive diode biased through a 1 megohm resistance monitors the fluorescence given of $f^{\prime}$. The diode generates a signal across the load resistor propurtional to the total cncrgy of the pulse and has a shape compatible with nuclear pulse shaping electronics. The light sensitive diode was found to have tremendous variation in sensitivity as a function of wavelength if $1 \mathrm{t}$ viewed the UV directly. Therefore, the fluorescent wavelength conversion was employed to improve the wavelength response. Nevertheless, the response was not wavelength independent and it was necessary to calibrate the detector as a function of wavelength. The voltage pulse across the diode load resistor is amplified and then digitized by a 100 channel TMC 401 pulse height 


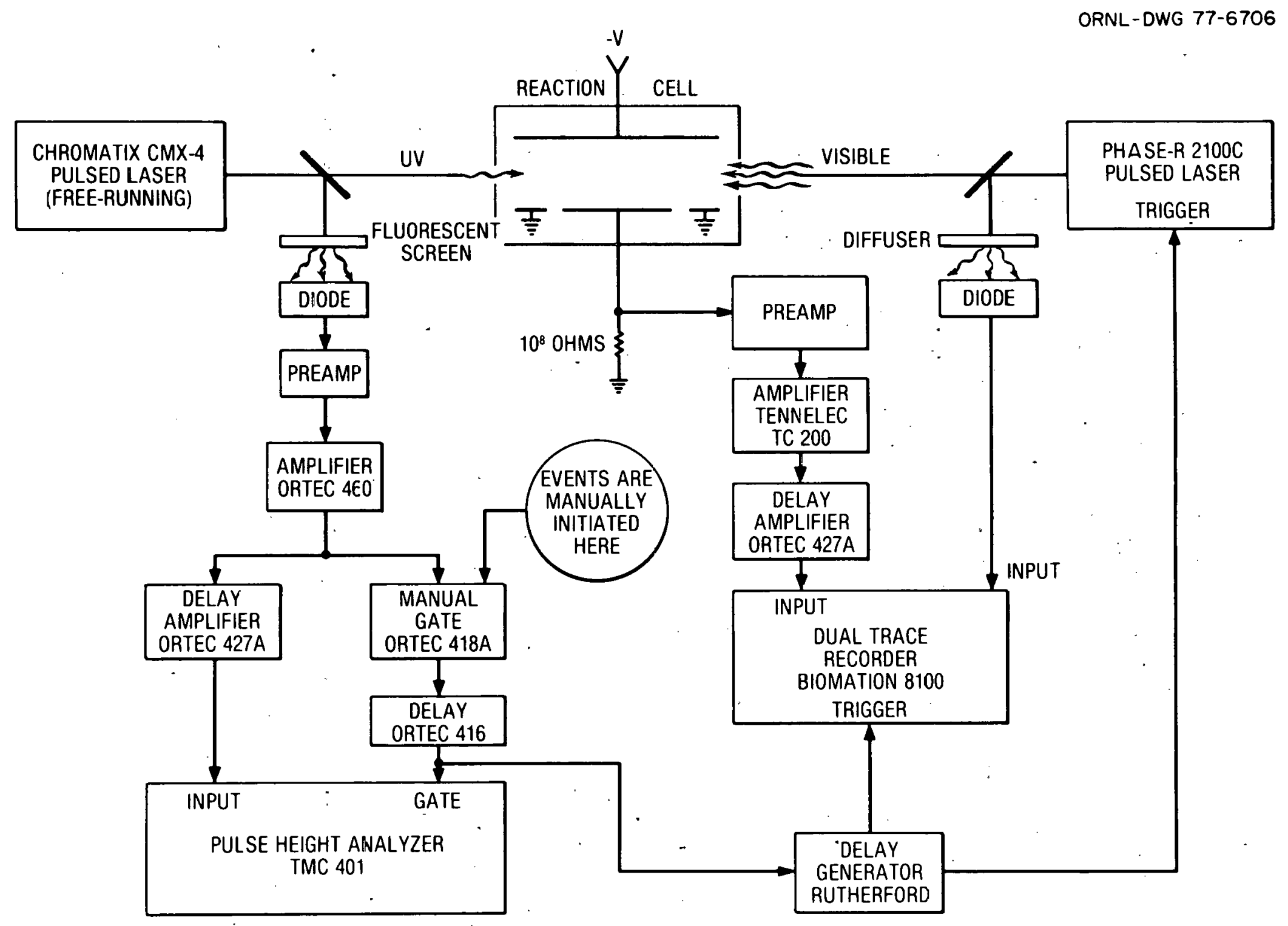

Figure 2. The data acquisition hardware. 
analyzer.

The source laser is free running at a repetition rate of 1 hertz: However, the ionization laser can be pulsed only about every 30 seconds. Therefore, an ortec $418 \mathrm{~A}$ coincidence gate is enabled by the operator once the detector laser is charged up and ready to flash. The next source diode pulse is gated into the memory of the TMC pulse height analyzer. This pulse also triggers the detector laser and a Biomation trace recorder through an electronic delay generator. Ionization produced in the guarded plate region of the reaction cell is amplified with a charge sensitive Ortec 109A preamplifier and Tennelec TC200 shaping amplifier. This system has an RMS noise of about 500 electrons. However, rf noise from the ionization laser raised the lower limit of detectability to about $3 \times 10^{4}$ electrons/pulse. The intensity of the detector laser is monitored with a beam splitter and light serisitive diode biased through 50 ohms. The signal generated across the resistor is recorded directly by the trace recorder. Both the diode pulse and the ionization pulse are recorded on a single uscllluscope trace using a differential amplifier input of the trace recorder. The ionization pulse is delayed with respect to the diode pulse with an Ortec $427 \mathrm{~A}$ delay amplifier. The address of the last source diode pulse stored in the PHA and the two pulses stored in the trace recorder are recorded by the operator. The PHA and trace recorder memories are cleared and, when the ionization laser is charged up, the next sequence is gated through.

Time delays are generated by a Rutherford digital delay generator. 
This unit is driven by an internal crystal oscillator circuit and it has a range of 0-1 sec in hundred nanosecond intervals. The accuracy of the various step-down counters of this unit was verified with a Tektronix 454 oscilloscope.

The diode light sensors were calibrated for absolute energy per pulse with ballistic thermopiles. The UV source diode was calibrated with a Quantronix 503 thermopile. This unit required a fast burst of approximately 100 pulses to produce a measurable reading. The reading was then compared to the distribution of pulses stored in the PHA. The detector diode was calibrated with both the Quantronix 503 and Hadron 108 thermopiles. Both manufacturers claimed calibration traceable to NBS standards but agreed only to within 15 percent.

\subsection{The Interaction Cell}

The interaction cell is a stainless steel chamber connected to a good vacuum system. When not in use the cell was maintained at $10^{-6}$ torr or less. At the bottom of the cell is a thin stainless steel cup. This cup is heated from underneath, outside the vacuum cell, by a copper block containing an electrical heater. Temperature of the cup is monitored with a Chromel-Alumel thermocouple mounted in the copper block next to the steel cup. The sample was prepared by placing a small chunk of CsI in the cup and heating the cup to melting point of CsI $(905 \mathrm{~K})$. The CsI melted and coated the inside surface of the cup. The normal operating temperature of the cup is $625 \mathrm{~K}$ which produces a CsI density in the laser beams of about $3 \times 10^{8} / \mathrm{cm}^{3}$ for most operating 
conditions. Al1 surfaces, except for the sample cup, were maintained at room temperature. It was found by measurement with a fine ChromelAlumel thermocouple that the temperature of the argon in the region of the laser beam was within 5 of $325 \mathrm{~K}$ for all pressures used in this study. The cell windows are quartz and are mounted on small extensions. The extensions reduce the chances that charge produced at the windows by the lasers will cause electric field changes in the guarded ionization region. The ionization plates are made of stainless steel with ceramic insulators. The ionization plate separation is $2.7 \mathrm{~cm}$. The guarded plate has a length of $4 \mathrm{~cm}$ and a width of $2.5 \mathrm{~cm}$. It is surrounded on all four. sides by a second plate of outside dimensions $6.2 \mathrm{~cm}$ by $4.7 \mathrm{~cm}$ to eliminate spurious edge effects. These two plates and the walls of the interaction cell are maintained at ground potential while the opposite plate is biased negative. With this arrangement all electrons created in the guarded fonization region can be collected by the guarded plate, while all electrons created by phuton interactions with the windows or the walls can be suppressed.

Matheson purity argon (99.9995\%) was used without additional purifying for the buffer gas. A flowing system was used to reduce possible impurities due to out-gasslng with an exchange ratc of once per two minutes. Measurements of oxygen reactions were done in a static system due to lack of a flow system capable of mixing the small percentages of oxygen (also Matheson purity). However, this was found to have no effect on the results. A Wallace and Tiernan SAl45 Vacuum gauge monitored the pressure inside the ionization cell. 
2.4 The Lasers

The ultraviolet source laser beam is obtained from a chromatix CMX-4 pulsed dye laser. This laser uses a linear flash lamp within an elliptical cavity. The flash lamp runs along one pole of the cavity and the dye cell along the other pole. A visible output of about 4 millijoules is produced in a $0.7 \mathrm{\mu sec}$ (FWHM) pulse. An ultraviolet output of about 0.1 millijoule is obtained with an intra-cavity doubling crystal. The visible output is removed with a band pass filter just beyond the output mirror. The laser beam diameter at the interaction cell is 3 millimeters. Some measurements are performed with a $50 \mathrm{~cm}$ focus quartz lens which produces a divergence limited beam of $0.05 \mathrm{~cm}$ diameter in the cell. When so focused, the laser produces a fluence in the cell greater than $10^{17}$ photons $/ \mathrm{cm}^{2}$.

The detector laser is a Phase-R $2100 \mathrm{C}$ pulsed dye laser. No tuning element is used inside the cavity; coarse tuning is accomplished by proper choice of dye and solvent. Lasing action occurs at the fluorescent peak of the mixture. When used in this manner, the laser has a bandwidth of $40 \AA$. Figure 3 shows two output spectra of the dye mixture used for this study $\left(1.5 \times 10^{-4}\right.$ molar coumarin 2 in $50 \% \cdot \mathrm{H}_{2} \mathrm{O}$ and 50\% methanol). The first spectrum is with fresh dye and pumps both the $\operatorname{Cs}\left(7^{2} \mathrm{P}_{3 / 2}\right)$ and $\operatorname{cs}\left(7^{2} \mathrm{P}_{1 / 2}\right)$ states. When both states are pumped, ionization saturation is easily achieved. After the dye has aged (i.e., used about 400 pulses), there is a shift to the red sufficient to prevent pumping of the $\operatorname{Cs}\left(7^{2} \mathrm{P}_{3 / 2}\right)$ level. The second spectrum indicates this condition. Saturation of ionization was checked for fresh and 
ORNL-DWG 77-6710R

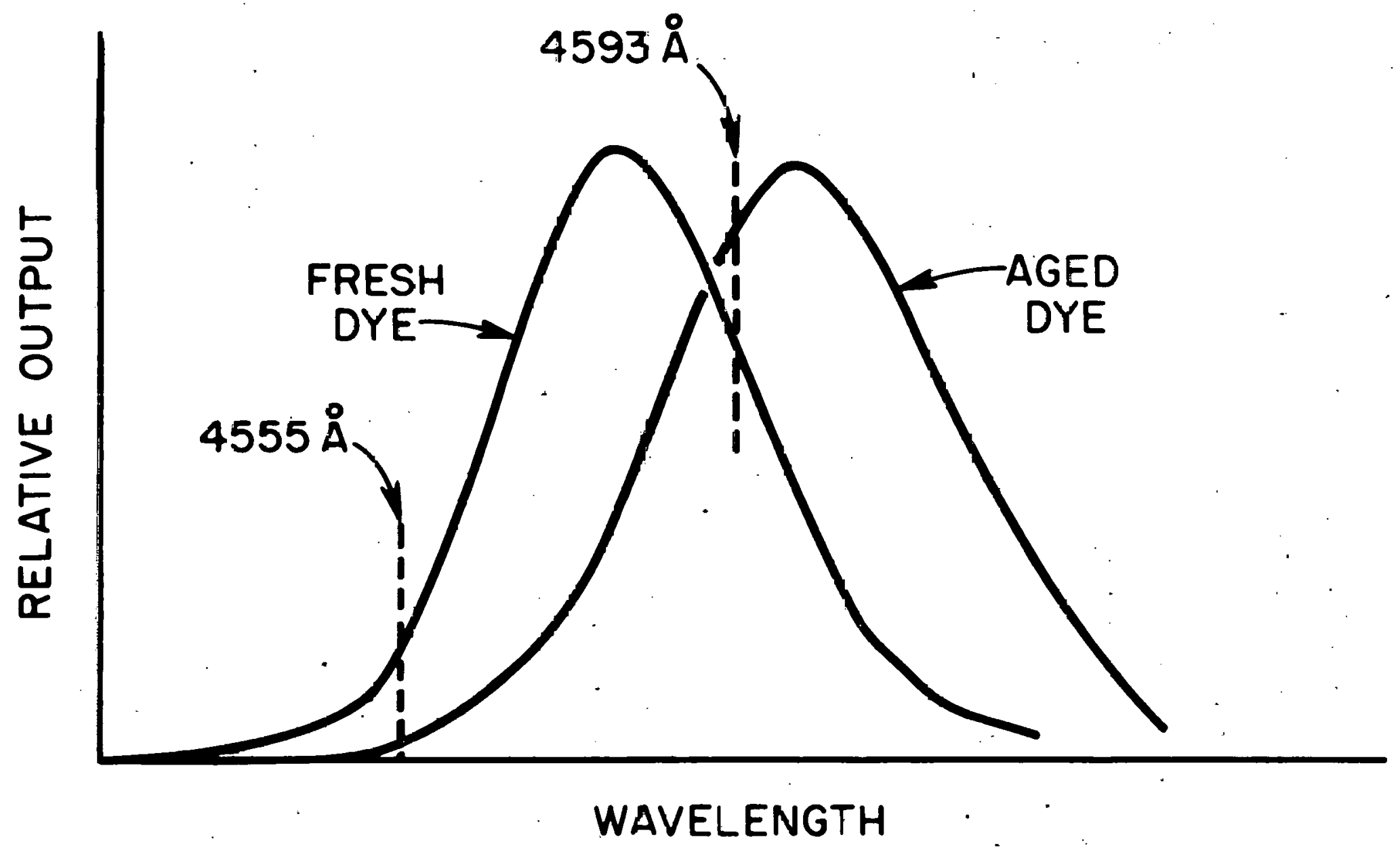

Figure 3. Relative intensity vs wavelength of a sirgle pulse of the detector laser. 
aged dye and the implications of these results will be discussed. When tuned to the $\operatorname{Cs}\left(7^{2} \mathrm{P}_{1 / 2}\right)$ leve1, 0.4 joule of laser energy is produced with a pulse width of $0.3 \mu \mathrm{sec}$. Lasing action is obtained by discharging 500 joules of electrical energy stored in a capacitor Marx Bank through the coaxial flash lamp surrounding the dye cell. The Marx Bank discharge is accomplished by breaking down a spark-gap switch with a high voltage pulse. The original spark gáp supplied by the manufacturer caused excessive delay between trigger pulse and breakdown. The spark gap was redesigned to include the center electrode of an automobile spark plug with a very successful result. To eliminate thermal gradients, which tend to spoil the cavity alignment, a triaxial cooling arrangement is employed. A closed cycle loop of cooling water is circulated through a hollow cylinder between the flash lamp and dye cavity. The dye is circulated through the heat exchange coils in the water reservoir just before entering the cavity. Thus, the lamp, water, and dye are maintained at the same temperature.

Output energy of a flash lamp pumped dye laser is roughly proportional to the flash lamp energy minus a threshold energy. The threshold is determined by many factors including type of cavity and the dye itself. For this experiment, threshold corresponded to 40 percent of the maximum rated energy of the lamp. It is not possible to pump the dye cavity uniformly. When viewed in cross section, various parts of the output beam have slightly different thresholds. Therefore, the intensity profile of the Phase- $R$ laser is most uniform at the highest pumping intensities of the flash lamp. When studying ionization 
yield as a function of intensity, the laser is maintained at maximum intensity. The output beam is then attenuated by a sertes of glass slides mounted slightly off-axis to the laser beam. Each slide surface reflects about four percent of the laser intensity and so twenty slides are required to reduce the beam intensity to twenty percent of its unattenuated value. Neutral density filters (e.g., glass absorption filters with aluminum coatings) are not used because of the likelihood of damage by $0.4 \mathrm{~J}$ pulses.

The output of the Phase-R laser is passed through a X2 doubleconvex beam reducing telescope. The beam diameter at the ionization cell is $(6.5 \pm 0.5) \mathrm{mm}$ and was determined by measuring burn patterns on carbon paper and developed polaroid film. 
SECTION 3

EXPERIMENTAL RESULTS AND DISCUSSION

\subsection{Photoionization of Cesium}

When an atom of cesium is irradiated with photons from a laser tuned to an optically allowed transition, absorption can occur. If one neglects all other processes, then an excited state so produced will decay by either spontaneous decay or stimulated emission. A high intensity laser pulse can bring a population of cesium atoms into an equilibrium where rate of excitation by absorption is the same as the rate of de-excitation. The rate of de-excitation by stimulated emission can be much larger than the rate of spontaneous decay. Thus, equilibrium can be established very rapidly during the course of the laser pulse and can exist throughout most of the time duration of the pulse. The ratio of the population of the excited state to the ground state is then simply the ratio of the degenerate magnetic substates of the two levels. It is assumed that excitation occurs in a buffer gas such that many collisions occur during a time comparable to the lifetime of the excited state and all memory of the excitation process is lost. Although no collisional depolarization data for the Cs $7^{2} \mathrm{P}$ levels has been found, much data exists for other alkali metal excited states. A depolarization cross section of $6 \times 10^{-15} \mathrm{~cm}^{2}$ for $\mathrm{Cs} 6^{2} \mathrm{P}$ level in 
argon ${ }^{17}$ seems to be typical. Such a cross section would make the degeneracy assumption quite valid at the pressures of this study. In order to establish equilibrium it is required that

$$
\int f(u) \sigma_{u 1}(u) d u>>\gamma_{u x}
$$

where $f(u)$ is the photon flux per unit frequency, $\sigma_{u l}$ is the cross section for stimulated emission and $\gamma_{\mathrm{ux}}$ is the spontaneous decay rate of the excited state. The Ionization laser is broadband so $f(u)$ is effectively a constant over the absorption integral. Furthermore, the we11 known Einstein formula for the integral of stimulated emission is given $\mathbf{a s}$

$$
\rho \sigma(u) d u=\frac{\lambda_{0}{ }^{2} \gamma_{u l}}{8 \pi},
$$

where $\lambda_{0}$ is the line center wavelength and $\gamma_{u l}$ is the spontaneous decay rate directly to the ground state. Substituting equation (2) into equation ( 1 ) and solving for $f(u)$ yields,

$$
f(u)>>\frac{8 \pi^{\gamma} u x}{\lambda_{0}^{2} \gamma_{u l}}
$$

According to Figure 4, spontaneous decay directly to the ground state is much less frequent than spontaneous decay through other channels, particularly the $6^{2} \mathrm{~S}$ levels. Also, the $40 \mathrm{~A}$ FWH bandspread of the detector laser is much broader than most pulsed lasers. Therefore, higher than expected photon fluences will be required to establish the equilibrium. Assuming a frequency bandwidth appropriate to $40 \hat{\mathrm{A}}$, wavelength spread centered at $4593 \AA$, and a laser pulse length of .5 usec, then equation (3) predicts that photon fluences much greater 


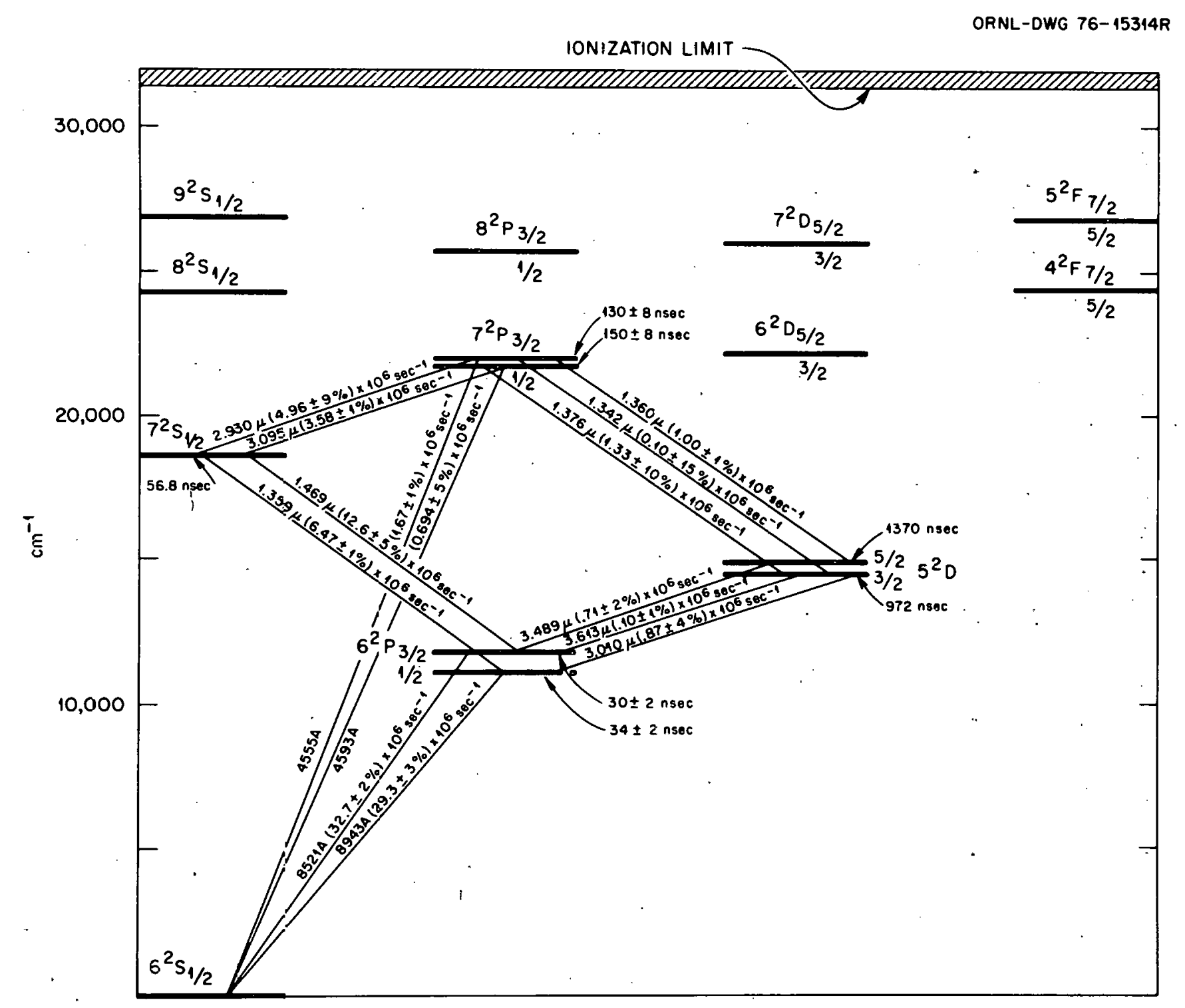

Figure 4. Spectrum wavelengths, transition rates and lifetimes for lower levels of the cesium atom. For the source material, see Ref. 4. Courtesy of Prof. Ray Hefferlin, presently at Oak Ridge National Laboratory on sabbatical from Southern Missionary College. 
than $100 \mathrm{~mJ} / \mathrm{cm}^{2}$ will be required to bring the $7^{2} \mathrm{P}_{1 / 2}$ level into equilibrium with the ground state.

Photoionization by $7^{2} \mathrm{P}$ resonance radiation can occur from any of the excited states populated by spontaneous decay of the $7^{2} \mathrm{P}$ levels. However, the lifetimes of all levels, except those of the $5^{2} \mathrm{D}$, are short and little effect on the ionization yield is expected. Lifetimes of the $5^{2} \mathrm{D}$ levels are approximately a microsecond and so considerable population of these levels might occur during a laser pulse. Manson, et al. 1.8 , calculate a photoionization cross section for the $7 p$ level (neglecting spin-orbit coupling) equal to $4.5 \times 10^{-18} \mathrm{~cm}^{2}$. Manson 19 also finds the cross section for photolonization of the $5 d$ to be $2.4 \times 10^{-17} \mathrm{~cm}^{2}$, a factor of 5 greater. Both cross sections are quoted for the resonance wavelength of the $7 p$ level. Therefore, at low intensities, a sizeable fraction of the ionization will come from the $5 \mathrm{D}$ levels. Also, the photon fluence required to make $\sigma \phi$ unity for the ip level corresponds to an energy density of approximately 100 $\mathrm{mJ} / \mathrm{cm}^{2}$. Therefore, the first excltation processes and the ionization require about the same energy per pulse. This is unusual and is chiefly a result of the broadband mode of operation of the detector laser.

Figure $\dot{j}$ is a voltage saturation curve showing the lonfzacton yield as a function of $E / P$ where $E$ is the electric field in the guarded plate region and $\mathrm{P}$ is the gas pressure. This was obtained at fixed intensities of source and detector lasers. This curve remained relative$1 y$ constant over the range of argon pressures used (25-400 torr). Over the flat portion of the curve, from 0.5 to 2.0 volts $-\mathrm{cm}^{-1}-\operatorname{torr}^{-1}$, all of 


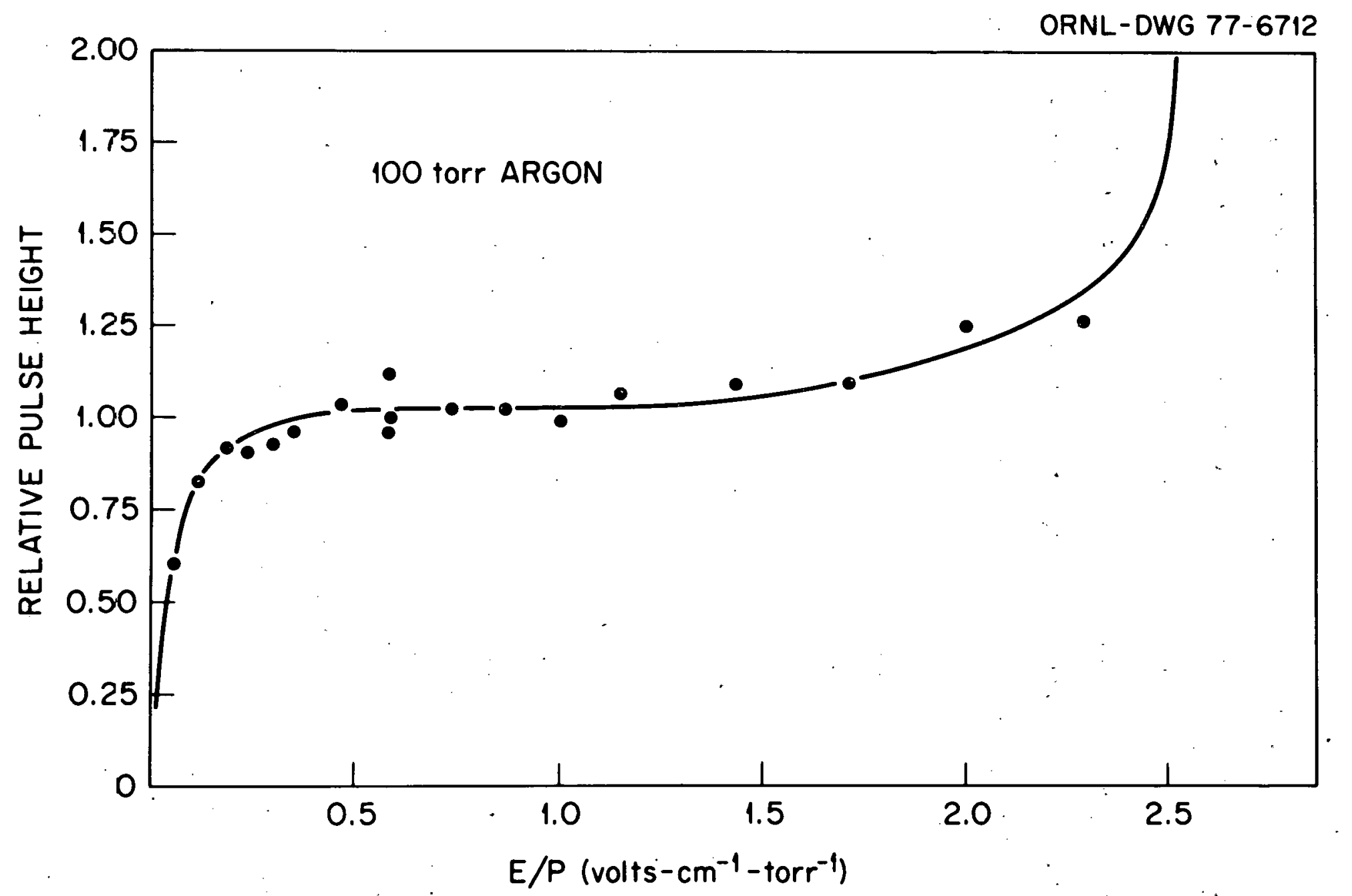

Figure 5. Voltage saturation curve of cesium in argon. 
the electrons created by the detector laser pulse are collected. At the lower vaues of $\mathrm{E} / \mathrm{P}$ there are losses due to recombination. At higher values of $\mathrm{E} / \mathrm{P}$, electron multiplication begins to occur. Also, voltage discharge will occur which saturates the electronics.

Operation of the ionization plates within the plateau region. assures the absence of space charge effects. If charge densities within the detector beam were high enough to cause space charge effects there would be no plateau. Care was taken to avoid space charge during al1 data acquisition.

Figure 6 shows ionisation yicld as a function of the detector laser's pulse intensity when fresh dye is used. This data is for a fixed intensity of the source laser. Strong saturation is seen with little dependence on the buffer gas pressure. Figure 7 shows ionization yield as a function of intensity after the dye has aged with several hundred pulses. Comparing the two figures, data taken at 100 torr show little dependence on the age of the dye. Data for 25 torr show that saturation requires a much higher intensity for the aged dye.

When the dye is fresh there is direct excitation of both the $\operatorname{cs}\left(7^{2} \mathrm{P}_{3 / 2}\right)$ and $\operatorname{cs}\left(7^{2} \mathrm{P}_{1 / 2}\right)$ levels. As the dye ages there is a slight spectrum shift toward longer wavelengths. The shift greatly decreases direct excitation of the $7^{2} \mathrm{P}_{3 / 2}$ level, as shown in Figure 3. However, the $7^{2} \mathrm{P}_{3 / 2}$ level can be populated by inelastic collisions with the argon buffer gas. The mixing is pressure dependent with a cross section of $1.2 \times 10^{-17} \mathrm{~cm}^{2}$ (ref. 20). Assuming a collisional velocity of $4 \times 10^{4} \mathrm{~cm} / \mathrm{sec}$, a mixing rate of $1.5 \times 10^{4} \mathrm{P}_{\operatorname{torr}^{-1}} \mathrm{sec}^{-1}$ is obtained 


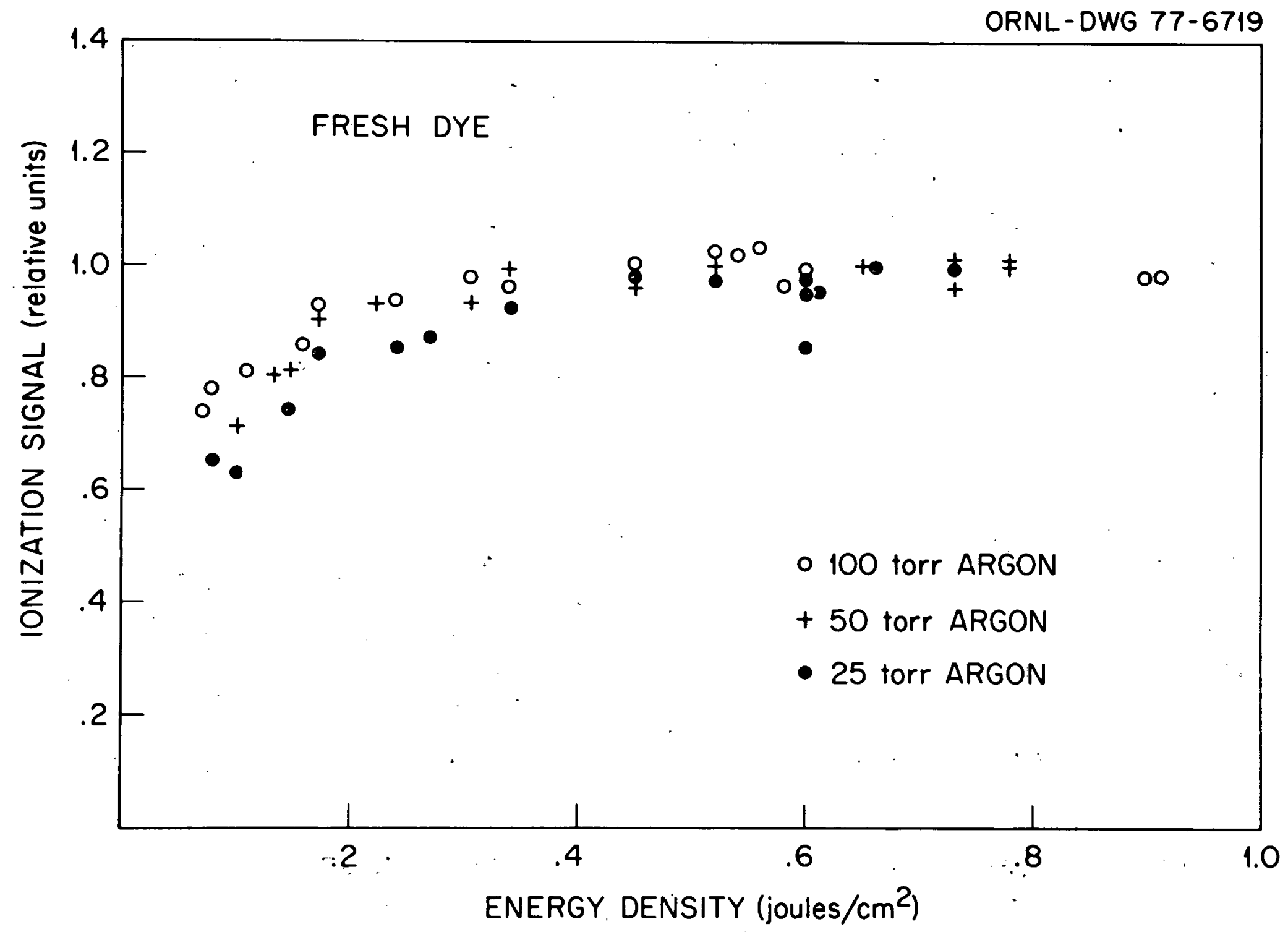

Figure 6. Iorization signal vs detector laser intensity using fresh dye. 


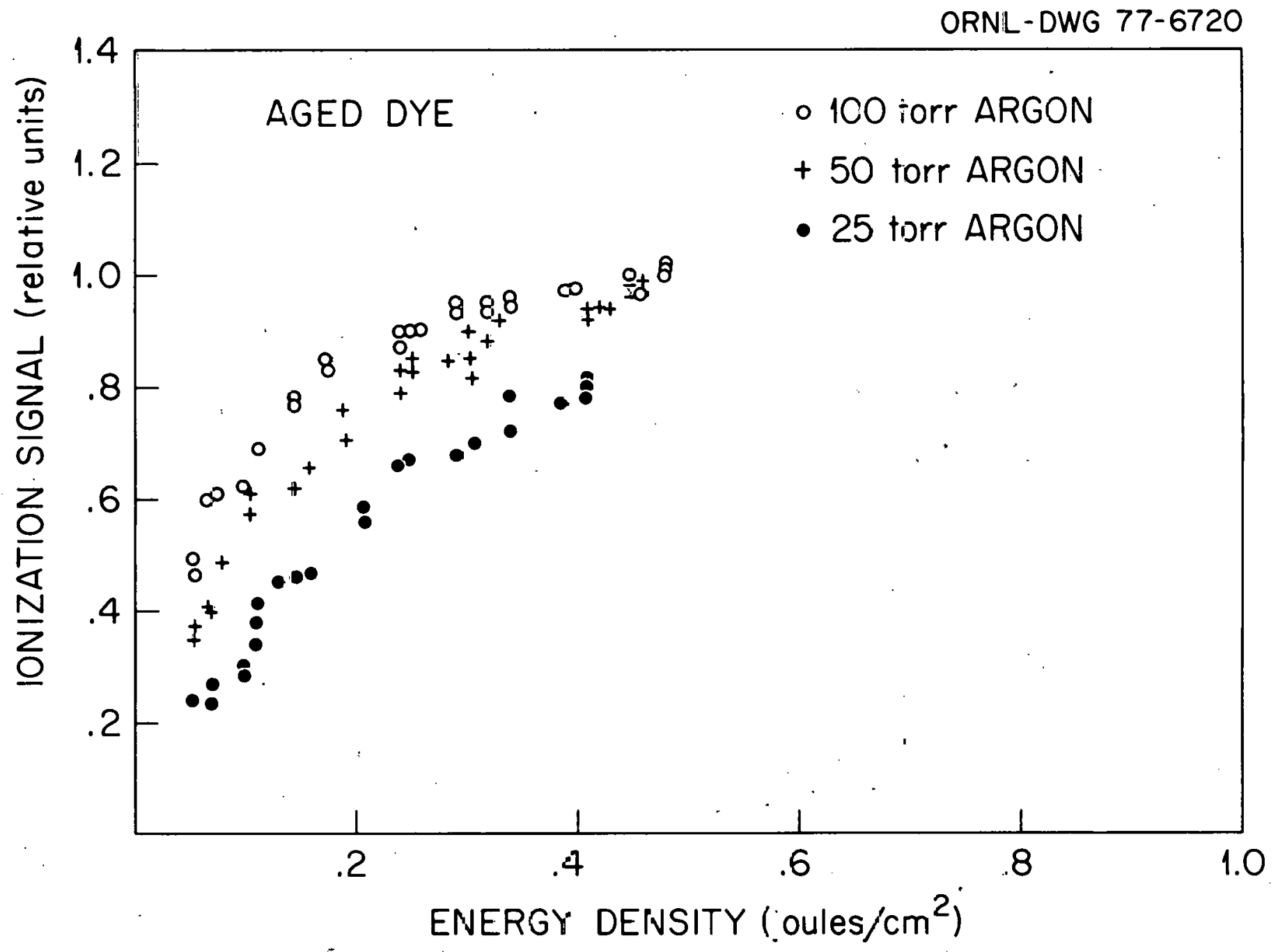

Figuze 7. Lonization sigral vs detector lase= intensity using aged dye. 
where $\mathrm{P}$ is the argon pressure. The product of rate and a pulse length of $.5 \mu \mathrm{sec}$ is unity at 220 torr. Collisional mixing of the $7^{2} \mathrm{P}$ and $6^{2} \mathrm{D}$ levels is possible. However, the cross section for this mixing is on the order of $1-3 \times 10^{-20} \mathrm{~cm}^{2}$ (ref. 20). Therefore, photoionization from the $6 \mathrm{~d}$ levels should be of no consequence. The Ionization saturation pressure dependence necessitated frequent dye changes when working at pressures below 100 torr.

Depopulation or quenching of excited states of alkali metals by molecular perturbers is well known to have large cross sections. Although no quenching data have been found for $\operatorname{Cs}\left(7^{2} \mathrm{P}\right)$, much data exists for the first excited states of many alkali metals. Typical values range from $10^{-15} \mathrm{~cm}^{2}$ to $10^{-14} \mathrm{~cm}^{2}$ depending on the gas and the alkali metals $21,22,23$. These cross sections would require unreasonably high concentrations of impurities in order to have an appreciable effect during the detector pulse. Accurate measurements of argon quenching have not been made, but in each case it was found the cross section was less than the lower limit of detectability $21,23$. Since the photoionization pressure dependence in Figure 7 is inverse to what one expects from quenching, it is felt that this experiment is. free of such difficulties.

Zeman 24 reports an experimental determination of the photoionization cross sections of the $7^{2} \mathrm{p}_{1 / 2}$ and $7^{2} \mathrm{P}_{3 / 2}$ levels with circularly polarized resonance radiation as $(6.2 \pm 0.5) \times 10^{-18} \mathrm{~cm}^{2}$ and $(8.8 \pm 1.6) \times 10^{-18} \mathrm{~cm}^{2}$, respectively. A dye laser was used in this experiment with pulses of $\frac{1}{2} \mu s e c$ duration. Cross sections were obtained 
by measuring the beam profile and then data fitting the non-linear. ionization yield as a function of intensity. It was felt that about 90 percent of the cesium was ionized at the maximum intensities. A spectfic accounting of the $5^{2} \mathrm{D}$ photoionization channel was not given. However, the results are in reasonable agreement with Manson's calculations. ${ }^{18,19}$. They do point out the need for accounting for spin-. orbit coupling.

An accurate determination of photoionization cross sections was not attempted in the present work because of several complications. Dual excitation of the $7^{2} \bar{P}_{1} / 2$ and $7^{2} P_{3 / 2}$ levels, as well as fallure to establish a discrete-discrete equilibrium at low intensities, are the main difficulties. At the expense of a reduction in peak intensities, dispersive elements placed in the lasing cavity produce much narrower bandwidth outputs. Then a single $\bar{j}^{2}$ p level could be excited and brought into equilibrium with the ground state. Since the dissociation laser has a beam diameter of only half that of the ionization laser, problems of beam non-uniformley at the edges are avolded. Cross scctions of both the $5^{2} \mathrm{D}$ levels and the $7^{2} \mathrm{P}$ levels at the wavelengths of the appropriate $7^{2} \mathrm{p}$. unpolarized resonance radiation could be obtained by modeling. Figure 6 indicates that some reduction In pulse intensity . could be tolerated without loss of accuracy.

\subsection{Saturated Photodissociation}

With sufficient fluence $\phi_{0}$ to saturate the ionization, 1.e., to detect each free atom in the laser beam, studies were made of the 
photodissoctation process. These studies were performed in 100 torr of argon with a source-to-detector time delay of $100 \mu \mathrm{sec}$. It was determined in the diffusion studies that losses due to diffusion or chemical reactions under these conditions were negligible. No ionization signals were observed due to the source laser alone. Berkowitz 25 observed ionization with a photoionization mass spectrometer only when the photon energy exceeded $7 \mathrm{eV}$.

Figure 8 shows the ionization signal as a function of the number of photons in a single pulse of the source laser, both for a focused and unfocused beam. Ionization yield is linear with intensity for the unfocused but showing a degree of saturation for the focused beam. The focused beam ionization does not reach an asymptotic value because of a non-uniform beam profile. The edges of the beam are not sharp. At high pulse intensities, a sizable fraction of the total number of dissociated atoms will come from the outer edge of the beam which never saturates.

To obtain the cesium photoproduction cross section, $\sigma$, the following analysis was made. A Gaussian beam is assumed for the source photon fluence $\sigma(\rho)$,

$$
\phi(\rho)=\phi_{0} e^{-\rho^{2} / R^{2}}
$$

where $\phi_{0}$ is the fluence when the radius $\rho=0$, and $R$ is a constant. The appropriateness of this assumption was roughly confirmed by direct photographs of a highly attenuated focused beam. The disşociation probability of a single molecule is assumed to be 


$$
P(\phi(p))=1-\exp (-\sigma \phi(\rho)) \text {. }
$$

The photoproduction yield per unit length of the laser beam $n_{I}$ is given by,

$$
\mathrm{n}_{\mathrm{I}}=\mathrm{N} \int_{0}^{\infty} 2 \pi \rho \operatorname{d\rho P}(\phi(\rho))
$$

where $\mathrm{N}$ is the number density of cesium iodide molecules. Substitution of equation (5) into equation (6) yields

$$
n_{I}=N \int_{0}^{\infty} 2 \pi \rho d \rho\left(1-\exp \left(-\sigma \phi_{0} e^{-\rho^{2} / R^{2}}\right)\right) \text {. }
$$

By using the variable substitution,

$$
v=v \psi_{0} e^{-p^{2} / R^{2}}
$$

and moving some constants to the left hand side, one obtains,

$$
\frac{n_{I}}{N \pi R^{2}}=\int_{0}^{\sigma \phi_{0}} \frac{d v}{v}\left[1-e^{-v}\right]
$$

The solution for this equation is given in incegral cables as

$$
F\left(u \psi_{0}\right)=\frac{n_{I}}{N \pi R^{2}}=\gamma i \ln \sigma \phi_{U}+E_{1}\left(\sigma \phi_{0}\right)
$$

where $E_{l}$ is the exponential integrai and $\gamma$ is Euler's constant

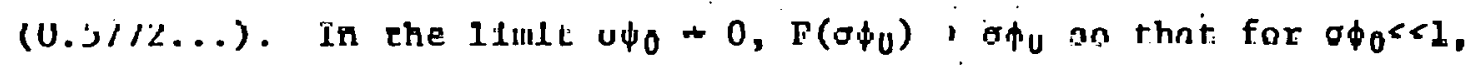

$$
\mathrm{n}_{\mathrm{I}}=\mathrm{N} \pi \mathrm{R}^{2} \sigma \phi_{0}=\mathrm{N} f \phi \mathrm{dA},
$$

the quantity $\pi R^{2} \phi_{0}$ is just the area integral of the Gaussian photon fluence. This asymptotic solution has the same linear dependence on the number of photons per pulse as the experimental unfocused beam data. Since the function $F\left(\sigma \phi_{0}\right)$ for $\sigma \phi_{0} \rightarrow U$ is $\sigma \phi_{0}$ itself, it is cunvenient to work with the ratio $F\left(\sigma \phi_{0}\right) / \sigma \phi_{0}$. Since the unfocused signal shows no sign of saturation, this ratio is just the ratio of the focused signal to the unfocused signal when both signals are measured at the same 


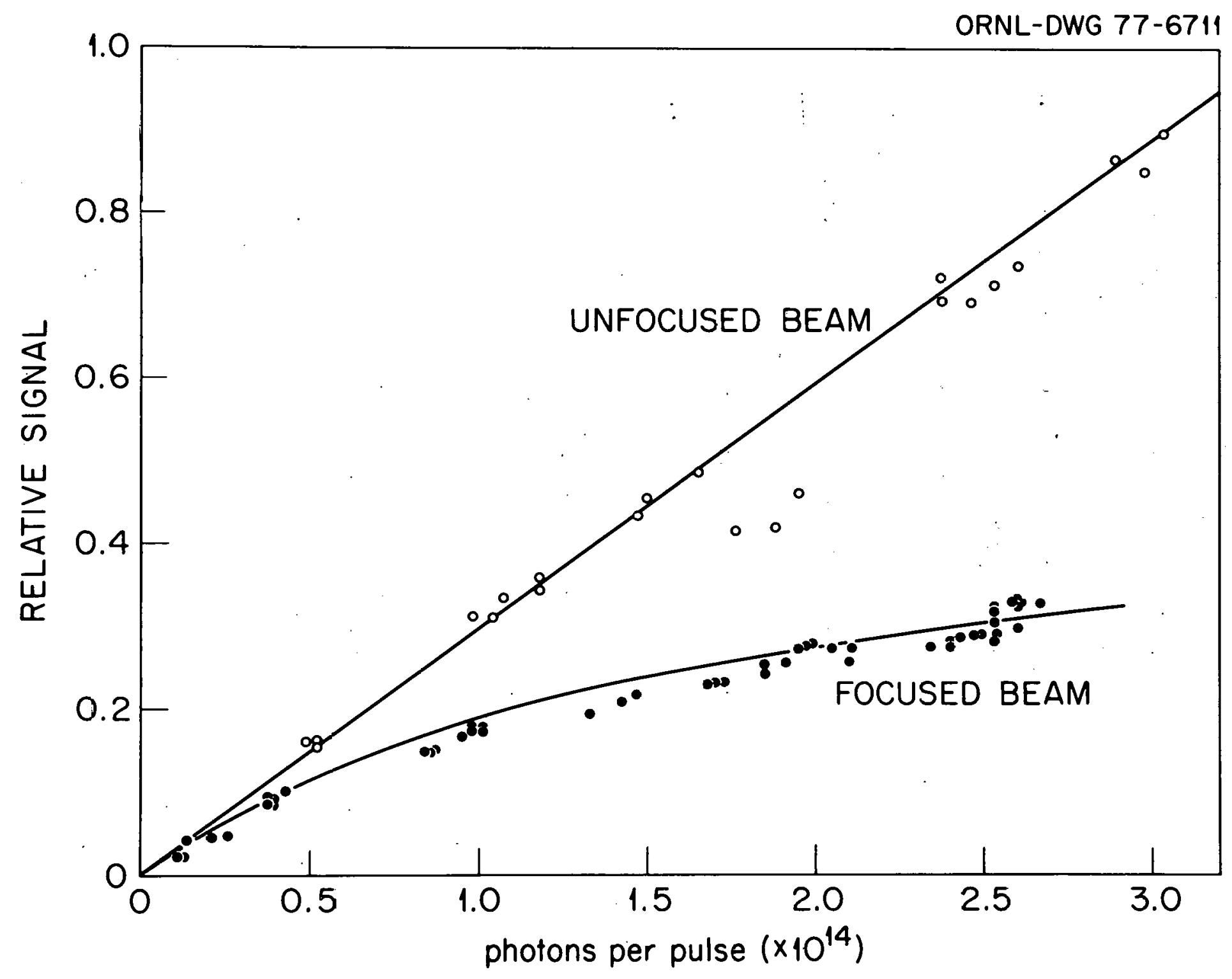

Figure 8. Ionization laser intensity vs photons per pulse of the source laser. 
number of photons per pulse.: For a given total number of photons (I used $2.5 \times 10^{14}$ since it falls within the largest cluster of data points), the value of $\sigma \phi_{0}$ was found that made $F\left(\sigma \phi_{0}\right) / \sigma \phi_{0}$ equal to the experimental ratio $(0.41)$. To complete the analysis for the cross section, $\phi_{0}$ must be determined. This was done by measuring the energy transmitted through a small pinhole of $0.25 . \mathrm{mm}$ diameter placed at the beam focus with a Molectron J3 pyroelectric Joulemeter. In this way, it was found at $3175 \stackrel{\circ}{A}$ the photoproduction cross section of cesium is $2.9 \times 10^{-17} \mathrm{~cm}^{2}$. The predicted (based on equation (8)) ionization yield of the focused snurce beam is shown as a omooth curve throigh the focused beam data of Figure 8 .

Figure 9 shows the cross section for the production of neutral cesium from CsI as a function of wavelength. The functional dependence was obtained hy sraling the ionization yleld yei plioton to that of $31,75 \mathrm{~A}$ data for an unfocused beam. The photoabsorption cross section determined at $1000 \mathrm{~K}$ (ref. 11) is shown in Figure 9 as a smooth curve. The present results lave a functional form which is slmilar to that of photoabsorption and agree to within a few percent of those of photoabsnrption at the peak. The present results are much narrower than the absorption data. This is attributed to the lower temperature used here $(325 \mathrm{~K})$ so that there is tar less populallun of the higher vibrational states of the CsI molecules 9 : With the present technique, a knowledge of the number density (or vapor pressure) of the CsI. molecules was not required. Results are obtained using signal strength in relative units. Also, an explicit determination of the Gauesian 


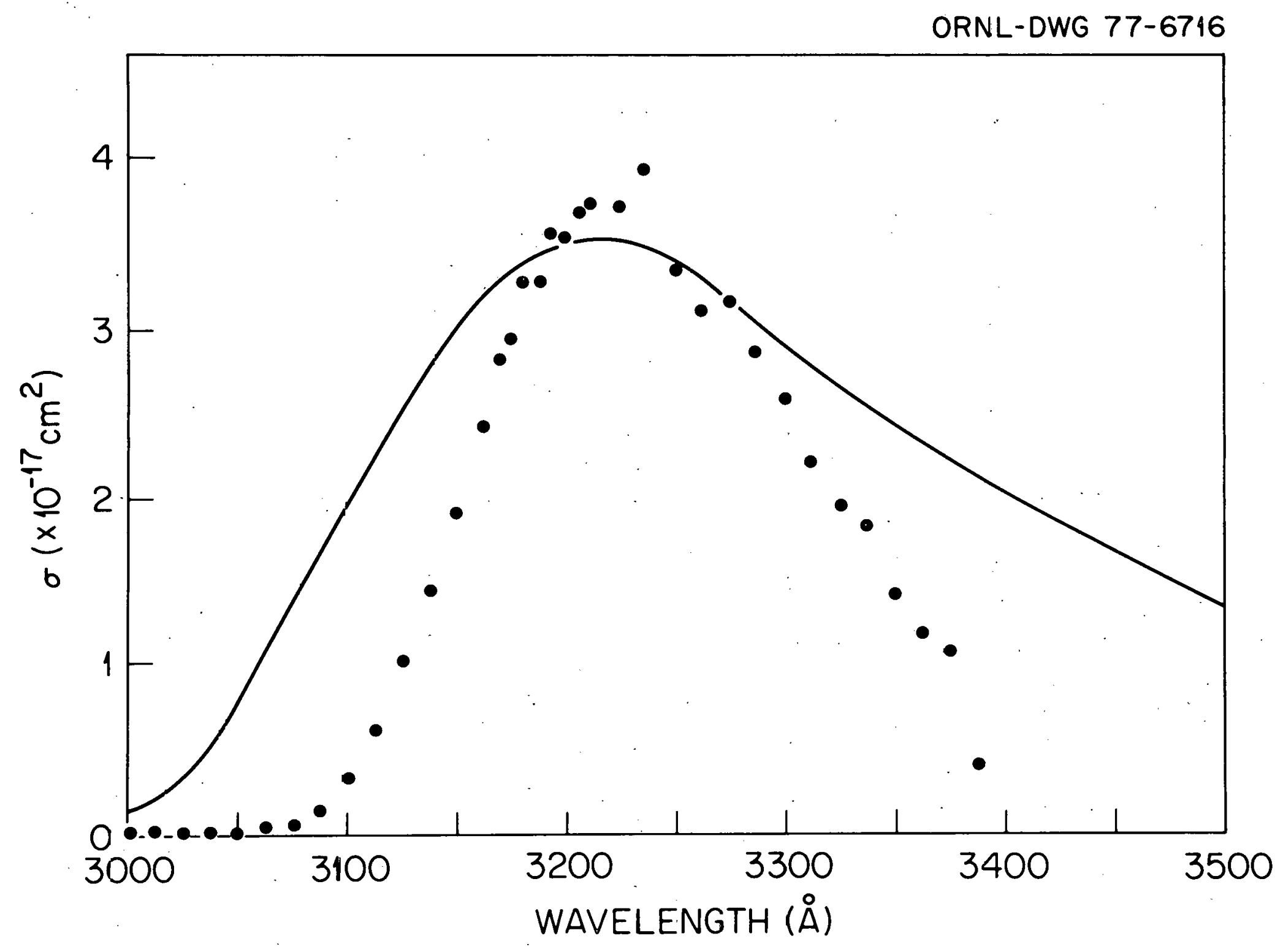

$\stackrel{\omega}{\omega}$

Figure 9. Cross section for the photoproduction of cesium from CsI as a function of wavelength. The photoabsorption data of Ref. 11 is shown as a smooth curve. 
parameter, $R$, is not required. of course, reasonably good agreement of the beam profile, $\phi(\rho)$, to equation (4) is required.

Alkali halide vapors are known to contain significant amounts of dimers and, in some cases, trimers 25-28. However, cestum halides have the least tendency to form dimers. Observable concentrations of cesium iodide dimers have not been found at $700 \mathrm{~K}$ using mass spectroscopic techniques 25, 27 . Also, Davidovits could see no evidence of CsI dimer absorption in the wavelength region of this study 11 .

Furthermore, our measurements were made at very much lower density of CsI than was previously possible, thus, in any event, our data chould be more free of dimer effects.

\subsection{Diffusion of Cesium in Argon}

Our experimental geometry (Figure 1 ) is idealiy suited to measurements of diffusion of alkali atoms in various atmospheres. Because of the well defined source and detector geometry, the effects of the apparatus wails can be entirely neglected. We proceed now to solve the diffusion equation appropriate to our measurements. At time, $t=0$, the cesium population $n(\vec{r}, t)$ is assumed to be

$$
n(\vec{r}, t)=\delta(x) \delta(y) \delta\left(z-z_{0}\right)
$$

The behavior of $n(\vec{r}, t)$ is simply the solution to the equation,

$$
\frac{\partial n(\vec{r}, t)}{\partial t}=D \nabla^{2} n(\vec{r}, t)
$$

subject to the Initial conditions of equation (9), where $D$ is the diffusion coefficient of the cesium in argon. It can be shown that the 
solution to equations (9) and (10) is,

$$
n(\vec{r}, t)=\frac{e^{-\left|\vec{r}-\vec{r}_{0}\right|^{2} / 4 D t}}{(4 \pi D t)^{3 / 2}}
$$

For an Infinitely long line along the $z$ axis having $\lambda$ dissociations per unit length at $t=0$, the population density $N(\rho, t)$ is given by,

$$
N(\rho, t)=\lambda \int_{-\infty}^{\infty} n\left(\left|\vec{r}-\vec{r}_{0}\right|\right) d z_{0}
$$

where $\vec{r}_{0}=\vec{k} z_{0}$ and $\rho=\sqrt{x^{2}+y^{2}}$. Substitution of equation (11) into equation (12) yields the expression,

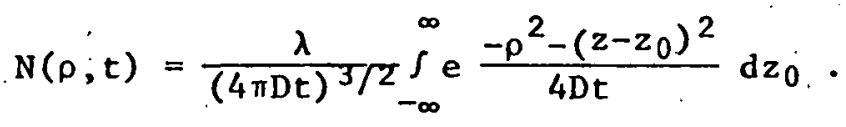

Performing the integration, the following result is obtained,

$$
N(\rho, t)=\frac{\lambda}{4 \pi D t} e^{-\rho^{2} / 4 D t}
$$

Therefore, the cesium population, $\mathrm{N}_{<_{R}}(t)$, contained within a cylinder of unit length and radius $R$ is given by

$$
N_{<R}(t)=\int_{0}^{R} 2 \pi \rho d \rho \lambda \frac{e^{-\rho^{2} / 4 D t}}{4 \pi D t} .
$$

Evaluating this integral, it is found that the fraction of the total population within the cylinder is given by,

$$
\frac{N_{<R}(t)}{\lambda}=1-e^{-R^{2} / 4 D t}
$$

For situations where the diffusing medium is not free of reactive components, then equation (10) becomes

$$
\frac{\partial n(\vec{r}, t)}{\partial t}=D \nabla^{2} n-\dot{B} n,
$$


where $B$ is the reactive decay constant.

The final solution for the fraction of atoms within a cylinder of radius $R$ at time $t$ is then given by

$$
\frac{\mathrm{N}_{<\mathrm{R}}}{\lambda}=\left(1-\mathrm{e}^{-\mathrm{R}^{2} / 4 \mathrm{Dt}}\right) \mathrm{e}^{-B \mathrm{t}}
$$

Experimental data was fit to relation (17) by an iterative process which assumed a value for $D$ and solved for $B$ at several time delays. D was varied until a value was found that gave the most consistent B's at the various delays.

Figure 10 shows data of the ionization signal as a function of time at 25 torn of argon. At very early times, few of the atoms have diffused far enough to escape the ionization cylinder. This produces an initial slow decay. Intermediate times have the most rapid decay but the rate of decay decreases and detectable signals are stili present at 0.3 seconds The smooth curve drawn through the points is the best fit obtained to equation (17) and represents $a=0$ and $D=0.11 \mathrm{~cm}^{2}$ $\sec ^{-1}$ at atmospherlc pressure. The curve deviates aomewhat from the data at very early times. This may be the result of a fallure to properly normalize the data at $t i m e, t=0$. The deviation at very large t1mes results from the finite dimensions of the gas cell, i.e., the medium is not truly unbounded. An estimate of the delay rime when llet wall effects should begin to play a role can be estimatcd by colving equation (17) for $t$ when $R=1.4 \mathrm{~cm}$, the distance from beam center to the ionization plates and $N / \lambda \doteq 0.90$. The solution is found to be $64 \mathrm{milliseconds}$ and, in fact, no departure of the data from the theoretical curve is seen 


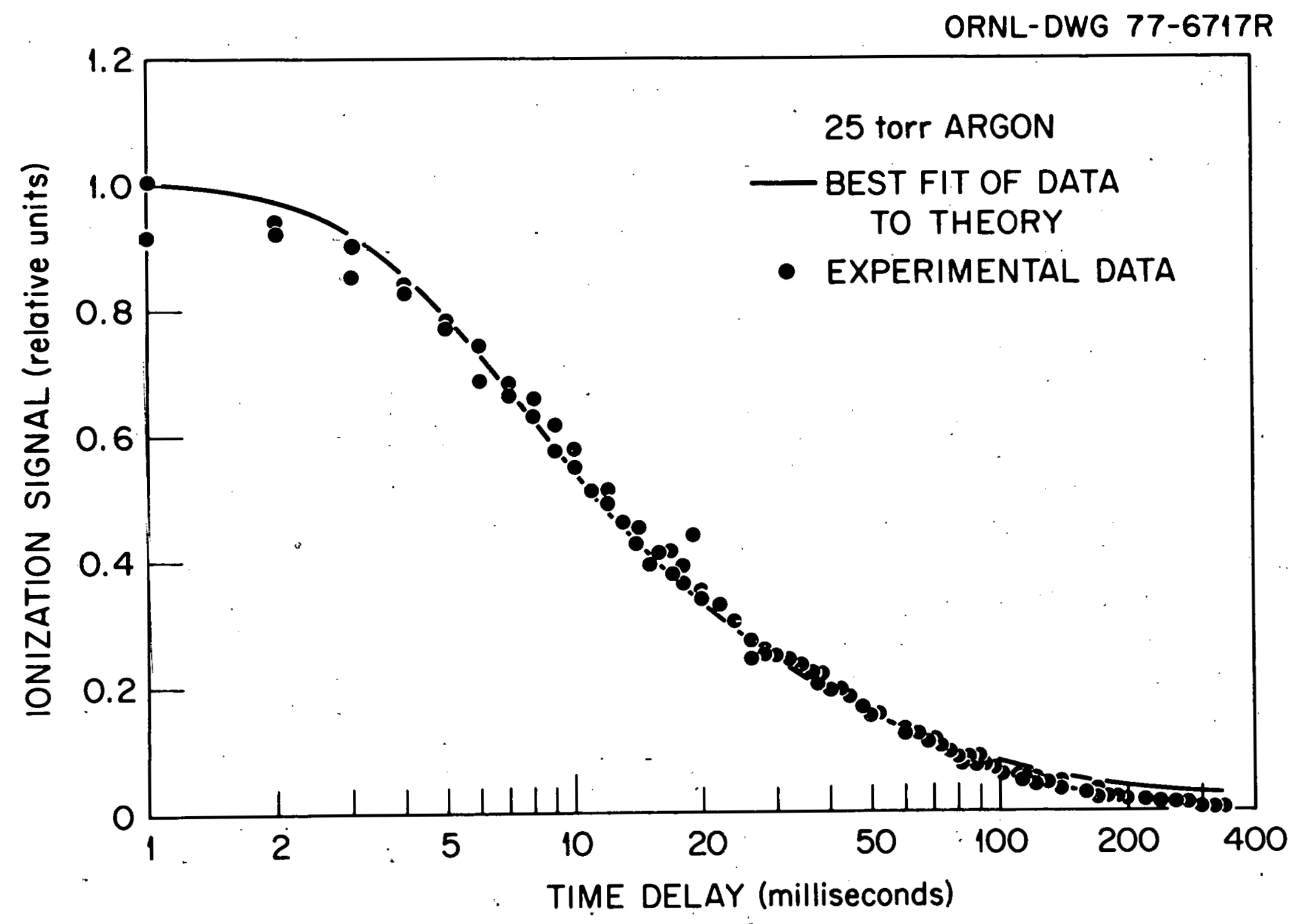

$\stackrel{w}{w}$

Figure 10." Relative cesium atom signal as a function of time in 25 torr of pure argon. 
until after this time.

Figure 11 shows ionization data taken at 50 torr of argon. Here the decay is slightly more rapid. This is contrary to the situation of pure diffusion, therefore, considerably faster reactive processes are involved at 50 torr compared to 25 torr. The best fit obtained to the data was $B=10.3 \mathrm{sec}^{-1}$ and $D=0.14 \mathrm{~cm}^{2}-\mathrm{sec}^{-1}$ at atmospheric pressure. A good fit with these parameters was obtained over the entire time scale. Wall effects do not play a significant role at late times at $5 n$ torr because reaction losses dominate the decay processes. The increasé in the palameter $B$ from 0 at 25 torr to $10 \mathrm{sec}^{-1}$ at 50 torr does not imply a threshold effect. Rather, at 25 torr the fitting of the decay is not sufficiently sensitive to changes in $B$ to allow $\ddot{a}$ better choice.

Additional data were taken at 100 torr and higher pressures. However, reaction processes are too fast to allow accurate determination of D. Also, there is an indication of other processes which may be associated with mass flow effects.

A mean value of $D$ for the two pressures studied is $0.12 \mathrm{~cm}^{2}-\mathrm{sec}^{-1}$ at atmospheric pressure. The two values agree to within 15 percent of this mean. Additional uncertainty was introduced by the edgen of the detector beam. The type of laser cavity combined with the telescope optics provided a reasonably sharp edge of the beam contour: Also, the beam was apertured just before entering the ionization cell. However, the ease of ionization with this intense pulse makes a knowledge of the beam radius more critical. The radius $R$ is felt to be determined to 


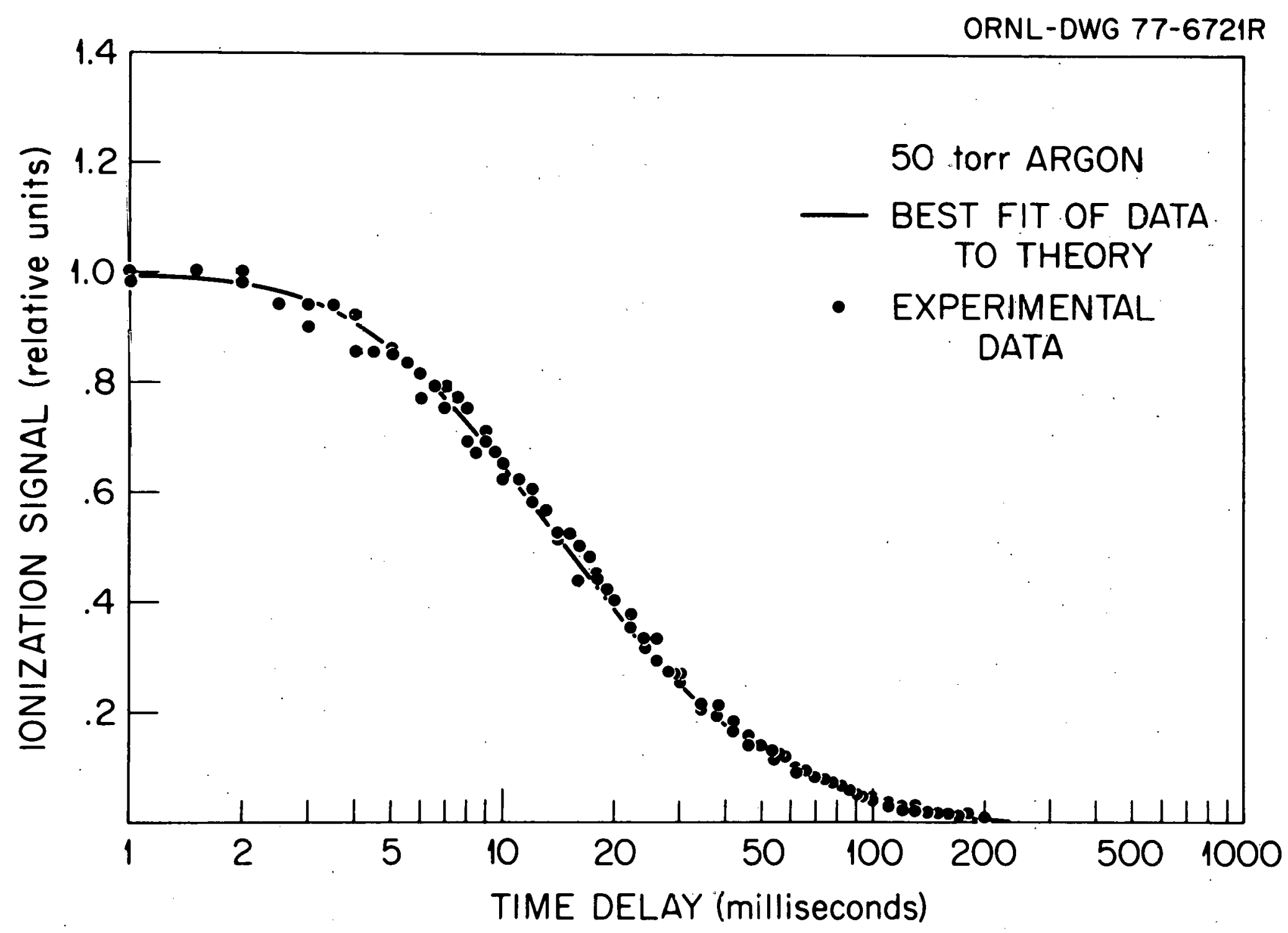

Figure 11. Relative cesium atom signal as a function of time in 50 torr of pure argon. 
within 10 percent which results in an uncertainty of 20 percent in $R^{2}$. Errors due to the finite dimensions of the cell should be small because they affect the data at very late times. Mass flow effects resulting from convection currents would tend to cause the estimate of $D$ to be too large. One suspects equation (17) would not provide a particularly good fit if mass flow difficulties were present. The fit is reasonably good so that for lack of evidence to the contrary it is assumed no such problem existed. It is hoped that uncertainty from other sources is small. If one assumes that the fitting procedure has an inherent uncertaincy equal lu the spread of tho two measurements, then this. combined with the uncertainty in $\mathrm{R}^{2}$, yields a total uncertainty about D. $=0.12 \mathrm{~cm}^{2}-\mathrm{sec}^{-1}$ of $\pm 25 \%$.

Directly comparable data for cesium in argon have not been found although there is data for somewhat similar systems. Franzen 29 measured values of $0.25 \mathrm{~cm}^{2}-\mathrm{sec}^{-1}$ for rubidium in argon and $0.31 \mathrm{~cm}^{2}-\mathrm{sec}^{-1}$ for rubidium in neon. Due to the increased atomic weight, diffuston coefficients of cesium should be smaller than those of rubidium for the same gas. However, it has been suggested that the technique used by Franzen provides only approximate results 29,31 . Accurate measurements have been made ot diffusion of trace amounta of kenun is argon 32 . These yield a D of $0.136 \mathrm{~cm}^{2}-\mathrm{sec}^{-1}$. Since cesium has an atomfc. weight very similar to that of xenon, a similar diffusion coefficient might be expected. 


\subsection{The Reaction of Free Cesium with Oxygen in an Argon Atmosphere}

The large signal to noise ratios combined with slow time decay of the cesium population in pure argon offered an excellent opportunity to study reactions of cesium with small amounts of an intentionally added impurity. Such studies were conducted with oxygen which is inert to cesium iodide but reacts vigorously with cesium. Figure 12 shows the effect of adding three small partial pressures of oxygen into 100 torr of argon. The ionization signal is controlled almost entirely by a simple exponential term over the range in time of Figure 12 . Losses due to other impurities or diffusion are negligible as shown by the uppermost curve. The slopes of the three oxygen curves yield the decay rates of $\beta=8 \times 10^{3} \mathrm{sec}^{-1}, \beta=1.4 \times 10^{4} \mathrm{sec}^{-1}$, and $\beta=2.3 \times 10^{4} \mathrm{sec}^{-1}$ for oxygen partial pressures of $0.01,0.02$, and 0.04 torr, respectively. These rates indicate a near linear dependence on oxygen concentration. Deviation of the rates from linearity is probably within experimental error as difficulty was encountered mixing the small percentages of oxygen used with the existing apparatus. Additional experiments conducted at 50 torr of argon revealed a linear dependence on argon pressure. An average of all the data at 50 torr and 100 torr results in a decay rate of $(7.5 \pm .7) \times 10^{-30} \sec ^{-1}-$ particle $e^{-2}-\mathrm{cm}^{6}$. The large uncertainty in the decay is a result of the difficulty in adding the small partial pressures of oxygen. As shown in Figure 12, the decay constants of a specific mix can be determined quite accurately.

Study at higher pressure was prevented by the sudden loss of the ionization signal. This is believed to be the result of electron 


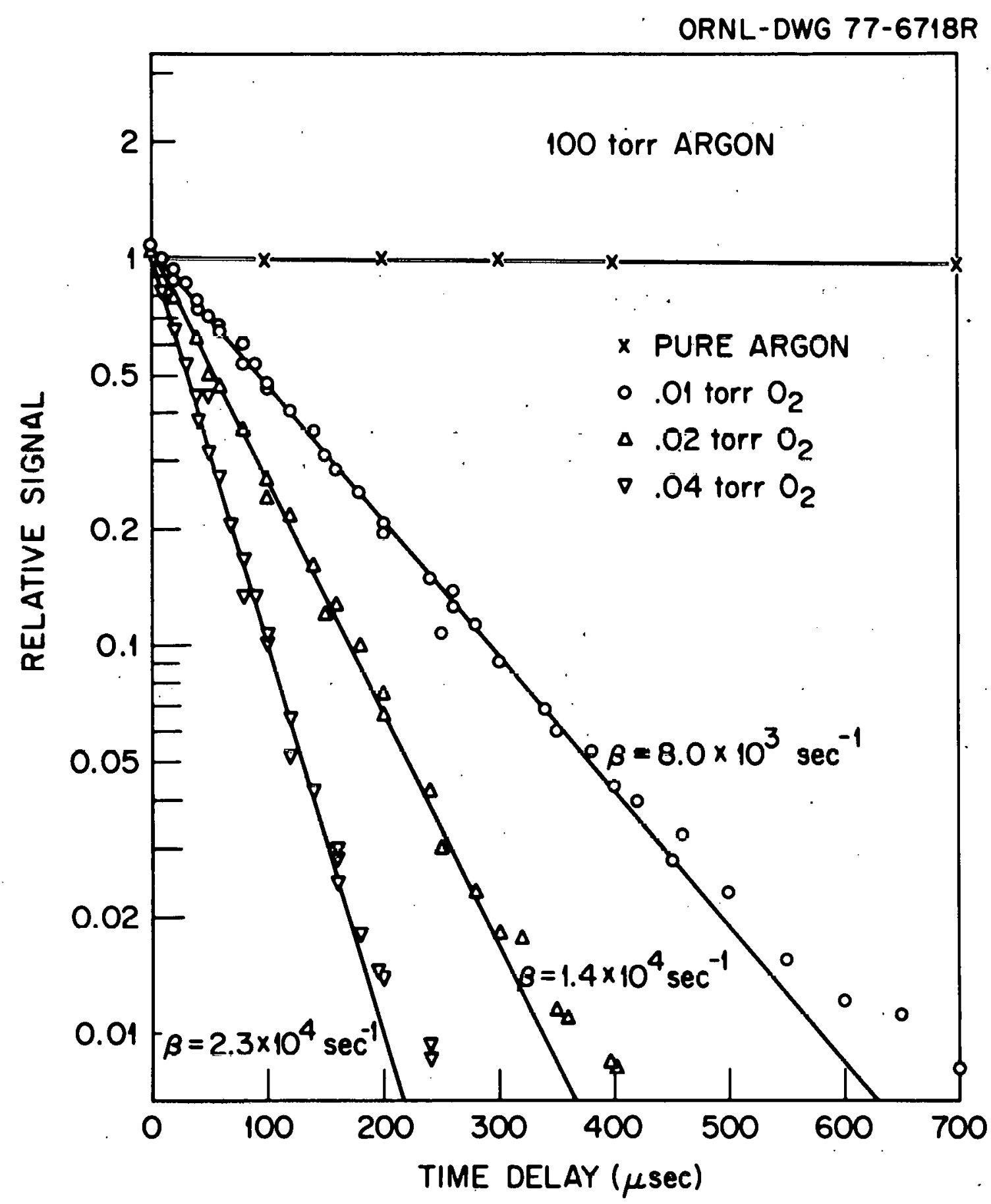

Figure 12. Relative cesium atom signal as a function of time for various partial pressures of oxygen in 100 torr of argon. 
attachment by oxygen molecules. Attachment studies ${ }^{33}$ of dilute. oxygen in argon mixtures show a strong tendency for the formation of heavy negative ions at the pressures and electric fields used in this study. The drift velocity of an electron is hundreds of times faster than that of a heavy negative ion. Once a free electron is attached to an oxygen molecule, the charge is essentially frozen in space, The preamplifier will not be able to detect any further change in voltage across the ionization plates from this electron. When a large fraction of the electrons are attached quickly, then rf noise of the Ionization laser pulse prevents accurate quantification of the reduced signal amplitude. The suddeness of the signal loss was surprising.

The linearity of the decay rate with both oxygen and argon implies that the reaction is associative, that is, cesium superoxide, $\mathrm{CsO}_{2}$ and not $\mathrm{CsO}$, is the reaction product. The argon buffer gas then serves as a stabilizing "third body" and the reaction is written in the following form:

$$
\mathrm{Cs}+\mathrm{O}_{2}+\mathrm{Ar} \rightarrow \mathrm{CsO}_{2}+\mathrm{Ar} \text {. }
$$

Since the superoxide is the stablest of the oxides at moderate temperatures 34 , no further reaction with oxygen is expected. The superoxide can react with water vapor and other impurities in the buffer gas. However, these rates will occur on a time scale similar to that of the diffusion studies.

Previous studies of the reactions of alkali metals with oxygen indicate that a three body rate is to be anticipated $35,36,37$. Measurements of the reaction of cesium and oxygen at temperatures com- 
parable to those of the present study have not been found: However, Carabetta and Kaskan 36 messured the reaction of cesium and sodium with oxygen in a hydrogen flame at $1500 \mathrm{~K}$. They used nitrogen as the third body and reported rates of $2.1 \times 10^{-33} \mathrm{sec}^{-1}-$ particle ${ }^{-2}-\mathrm{cm}^{6}$ for. cesium and $.82 \times 10^{-33} \mathrm{sec}^{-1}$-particle $\mathrm{e}^{-2}-\mathrm{cm}^{6}$ for sodium. Bawn and Evans ${ }^{35}$ measured the reaction of sodium and oxygen in nitrogen at $600^{\circ} \mathrm{K}$ using a diffusion flow technique. They report a rate of $2.2 \times 10^{-30} \mathrm{sec}^{-1}-$ particle ${ }^{-2}-\mathrm{cm}^{6}$. At pressures greater than 10 torr of nitrogen, the reaction rate failed to increase linearly with nitrogen pressure. This was interpreted as an approach to the stabilization of all the superoxide molecules within their lifetimes. Therefore, the reaction rate was undergoing a transition of character from termolecular to bimolecular. By curve fitting, the bimolecular reaction rate and lifetime of the unstabilized sodium superoxide were estimated to be $3.3 \times 10^{-12} \mathrm{sec}^{-1}$-particle ${ }^{-1}-\mathrm{cm}^{3}$ and $2 \times 10^{-9} \mathrm{sec}$.

In view of the results of Bawn and Evans ${ }^{35}$, it is tempting to speculate on the possibillty of observing similar results for cesium with the present apparatus. Consider the reactions:

$$
\begin{array}{ll}
\mathrm{Cs}+\mathrm{O}_{2} \rightarrow(\mathrm{CsO})^{-} & \mathrm{k}_{1} \\
\left(\mathrm{CsO}_{2}\right)^{-} \rightarrow \mathrm{Cs}+\mathrm{O}_{2} & \mathrm{k}_{2} \\
\left(\mathrm{CsO}_{2}\right)^{-}+\mathrm{A} \rightarrow \mathrm{CsO}_{2}+\mathrm{A} & \mathrm{k}_{3}
\end{array}
$$

where C.s, $\mathrm{O}_{2}$ and $\mathrm{A}$ are the concentrations of the reartants and the $k^{\prime} s$ are the reaction rate constants. The rate equations for the above 
system are,

$$
\begin{aligned}
& \frac{d(\mathrm{Cs})}{d t}=-k_{1}(\mathrm{Cs})\left(\mathrm{O}_{2}\right)+\mathrm{k}_{2}\left(\mathrm{CsO}_{2}\right)^{\prime} \\
& \frac{\mathrm{d}\left(\mathrm{CsO}_{2}\right)}{d t}=\mathrm{k}_{1}(\mathrm{Cs})\left(\mathrm{O}_{2}\right)-\mathrm{k}_{2}\left(\mathrm{CsO}_{2}\right)^{-}-\mathrm{k}_{3} \mathrm{~A}\left(\mathrm{CsO}_{2}\right)^{-}
\end{aligned}
$$

The reciprocal lifetime of the unstabilized superoxide, $k_{2}$, may be quite fast compared to the rate of formation of the superoxide. If this is the case, then the population of $\left(\mathrm{CsO}_{2}\right)^{-}$will be much less than that of Cs. Also, the time derivative of $\left(\mathrm{CsO}_{2}\right)^{-}$will be small compared to that of Cs. Therefore, an approximate solution can be obtained if equation (18b) is set equal to zero. Then,

$$
\left(\mathrm{CsO}_{2}\right)^{\prime}=\frac{k_{1}(\mathrm{Cs})\left(\mathrm{O}_{2}\right)}{k_{2}+k_{3} \mathrm{~A}} .
$$

Substitution into equation (18a) yields,

$$
\begin{aligned}
\frac{d(C s)}{d t} & =-k_{1}(C s)\left(O_{2}\right)+\frac{k_{2} k_{1}(C s)\left(O_{2}\right)}{k_{2}+k_{3} A} \\
& =-k_{1}(C s)\left(O_{2}\right)\left(1-\frac{k_{2}}{k_{2}+k_{3} A}\right),
\end{aligned}
$$

which is always less than the rate of formation of the unstabilized superoxide. When $\mathrm{k}_{2} \gg \mathrm{k}_{3} \mathrm{~A}$, then the fractional term in equation (20) can be expanded. Keeping the first two terms of the expansion one has,

$$
\begin{aligned}
\frac{d(C s)}{d t} & =-k_{1}(C s)\left(O_{2}\right)\left(1-1+k_{3} A / k_{2}\right) \\
& =-\frac{k_{1} k_{3}}{k_{2}}(C s)\left(O_{2}\right) A .
\end{aligned}
$$


Here one obtains a three body rate linear in oxygen and argon concentrations:

The "harpooning model" is the simplest theory which predicts reaction cross sections resulting in ionic bonding 38,39 . For the case of $\mathrm{CsO}_{2}$ the harpooning model provides an estimate of the bimolecular rate of forming the unstabilized superoxide. Studies of alkali- $\mathrm{I}_{2}$ and alkali-Br 2 reactions, which are dissociative and independent of buffer gas pressure, give results agreeing to within a factor of two of the harpooning model 13, 14. In this theory, the reaction cross section is simply $\pi r_{c}^{2}$ where $r_{c}$ is the distance for the crossing of the zeroth. order ionic and covalent curves. This distance. is given by

$$
r_{c}=e^{2} /\left(I(C s)-E\left(O_{2}\right)\right)
$$

where $I(\mathrm{Cs})$ is the ionization potential of cesium and $\mathrm{E}\left(\mathrm{O}_{2}\right)$ is the electron affinity of the oxygen molecule. Therefore, assuming an electron affinity of $0.45 \mathrm{eV}^{40}$, a reaction cross section, $\sigma$, of approximately $50 A^{2}$ is obtained. Assuming a collision velocity of $4 \times 10^{4} \mathrm{~cm} / \mathrm{sec}$ and an oxygen partial pressure of 0.01 torr (the smallest partial pressure of Figure 12), the bimolecular rate for the formation of $\left(\mathrm{CsO}_{2}\right)^{\prime}$ is given by

$$
\begin{aligned}
B & =\sigma N v \\
& =7 \times 10^{4} \mathrm{sec}^{-1} .
\end{aligned}
$$

This is only ten times the observed three-body decay of Figure 12. Therefore, the ratio $k_{3} A / k_{2}$ as contained in equation (21) could be approximately 1/10. Increasing the argon pressure a factor of ten 
might invalidate the three body decay rate and allow an estimation of $k_{1}$ to be made. The chief obstacle to such a measurement is the problem of electron attachment. At 1000 torr of argon, ionization signals might be quite small and require special effort to eliminate large uncertainties due to the laser rf noise transient. 


\section{SECTION 4}

\section{SUMMARY}

Study of the ionization yield as a function of detector pulse intensity indicates the process can be strongly saturated, that is, all of the cesium atoms within the detector beam can be ionized. Study of the dissociation process with a focused and thus extremely intense source pulse indicates that in the central portion of the beam this is also saturated. Thése resultes, combined with a previously demonstrated ability to detect single atoms of cesium, prove the ability to detect single molecules of cesium iodide.

Saturation of the dissoctation process provides a measure of the cesium iodide concentration. By studying relative ionfation as a function of photons per pulse of the dissociating laser, and by accounting for the laser beam intensity profile, it was possible to determine the absolute photodissociation of cesium iodide into its ground-state atomic consttuents. Knowledge of the vapor priessure of cesium iodide was not required, thus obviating the task of constructing an isothermal saturated vapor pressure cell.

The dissociation/ionization technique has an excellent signal-tonoise ratio even in a simple parallel plate ionization chamber. By saturating the lonfzation, signal dependence on butter gas pressure or detector laser intensity is eliminated. Furthermore, the ionization pulse 
amplitude is linearly related to the absolute number of cesium atoms in the ionization beam. Therefore, the decay in number of free cesium atoms within the ionization beam can be followed for many orders of magnitude.

Using a tightly focused source laser, free cesium atoms were created along the axis of the ionization beam. The functional form of diffusion transport out of the ionization volume, accounting also for reactions with impurities, is easily calculated. Diffusion transport was found to dominate the decay of cesium atoms out of the ionization column at argon pressures below 100 torr. The extraction of a diffusion coefficient was then accomplished rather directly by data fitting. The diffusion coefficient of cesium in argon was found to be $0.12 \mathrm{~cm}^{2}-\mathrm{sec}^{-1} \pm 25 \%$ at atmospheric pressure. More conventional techniques for the determination of diffusion coefficients require the gaseous components to be inert and noncondensing at the container walls. However, in the present study, this requirement was relaxed because diffusion was observed on a millisecond time scale far from the walls of the container.

The reaction of cesium with oxygen in the atmosphere of an argon buffer gas was studied. Oxygen pressures were selected so that the resulting reactive decay occurred on a microsecond time scale. Diffusion processes then had a negligible influence on the time dependence of the cesium decay. A three-body reaction rate of $(7.5 \pm 0.7) \times 10^{-30} \mathrm{sec}^{-1}$ particle $\mathrm{cm}^{6}$ was determined. This rate is 
appropriate to argon pressures as high at 100 torr. The ionization volume temperature was $325 \mathrm{~K}$. 
SECTION 5

REFERENCES

1. G.S. Hurst, M.G. Payne, M.H. Nayfeh, J.P. Judish and E.B. Wagner, Phys. Rev. Lett. 35, 82 (1975).

2. M.G. Payne, G.S. Hurst, M.H. Nayfeh, J.P. Judish, C.H. Chen, E.B. Wagner and J.P. Young, Phys. Rev. Lett. 35, 1154 (1975).

3. G.S. Hurst, M.H. Nayfeh and J.P. Young, Appl. Phys. Lett. 30, 229 (1977).

4. G.S. Hurst, M.H. Nayfeh and J.P: Young, "One-Atom Detection Using Resonance Ionization Spectroscopy," Phys. Rev. A, in press.

5. F.M. Lussier, Laser Focus 13 , No. 2; 48 (1977).

6. C.W. Choi, Dissertation, University of Kentucky (1977).

7. K. Sommermeyer, Z. Phys: 56, 548 (1929).

8. H.D. Schmidt-Ott, Z. Phys, 69, 724 (1931).

9. R.F. Barrow, A.D. Caunt, Proc. Roy. Soc. (London) A219, 120 (1953).

10. R.S. Berry, W. Klemperer, J. Chem. Phys. 26, 724 (1957).

11. P. Davidovits, D.C. Brodhead, J. Chem. Phys. 46, 2968 (1967).

12. A. Terenin, Z. Phys. 37, 98 (1926).

13. S.A. Edelstein, P, Davidovits, J. Chem. Phys. 55, 5164 (1971).

14. J. Maya, P. Davidovits, J. Chem. Phys. 59, 3143 (1973).

15. For an excellent review see: W. Jost, Diffusion in Solids, Liquids and Gases, (Academic Press, Inc., New York, 1960), Chapter $\mathrm{X}$.

16. T.N. Bell, I.R. Schankland and P.J. Dunlop, Chem. Phys. Lett. 45, 445 (1977). 
17. J. Fricke, J. Haas, E. Luscher and F. A. Franz, Phys. Rev. 163, 45 (1967).

18. S. Manson, A. Msezane, R.F. Reilman, Private Communication.

19. A. Msezane, S. Manson, Phys. Rev. Lett. 35, 364 (1975).

20. J. Cuvellier, P.R. Fournier, F. Gounand, J. Pascale and J. Berlande, Phys. Rev. A 11, 846 (1975).

21. D.R. Jenkins, Proc. R. Soc. A303, 453 (1968).

22. J.R. Barker, R.E. Weston, J. Chem. Phys. 65, 1427 (1976).

23. S. Lin, R.E. Weston, J. Chem. Phys. 65, 1443 (1976).

24. H.D. Zeman, "Electron Spin Polarization from Multiple Photoionization." in International Symposium on Electron and Photon Interactions with Atoma; cd. II. Kleinpoppen and M.R.C. MeDuwell (Plenum Press, New York, 1974), pp. 581-594.

25. J. Berkowitz, J. Chem. Phys. 50, 3503 (1969).

26. R.C. Miller, P. Kusch, J. Chem. Phys. 25, 860 (1956).

27. J. Berkowitz, W.A. Chupka, J. Chem. Phys. 29, 653.(1958).

28. S. Datz, W.T. Smith and E.H. Taylor, J. Chem. Phys. 34, 558 (1961).

29. W. Franzen, Phys. Rev. 115, 850 (1959).

30. L.W. Anderson, A.'l'. Kamsey, Khys. Kev. 132, 712 (1963).

31. A.L. Bloom, Yhys. Kev. 118, 664 (1960).

32. I. Amdur, 'I.F. Schatzki, J. Chem. Phys. 27, 1049 (1947).

33. T.E. Bortner, G.S. Hurst, Health Phys. 1, 39 (1958).

34. T.P. Whaley, Comprehensive Inorganic Chemistry, Vol. T, ed. by

J.C. Bailar,Jr., et al. (Pergamon Press, New York, 1973), p. 417.

35. C.E.H. Bawn, A.G. Evans, Trans. Faraday Soc. 33, 1580 (1937).

36. R. Carabetta, W.E. Kaskan, J. Phys. Chem. 72, 2483 (1968).

37. M.J. McEwan, L.F. Philips, Trans. Faraday Soc. 62, 1717 (1966).

38. J.L. MaGee, J. Chem. Phys. 8, 687 (1940). 
39. D.R. Herschbach, Adv. Chem. Phys. 10, 319 (1966).

40. F.M. Page, "Electron Affinities," in CRC Handbook of Chemistry and Physics, 56th Edition, ed. by R.C. Weast (CRC Press, Cleveland, 1975), p. E67. 


\section{THIS PAGE \\ WAS INTENTIONALLY \\ LEFT BLANK}


ORNL/TM-6014

\section{INTERNAL DISTRIBUTION}

$\begin{aligned} & \text { 1. } \text { Biology Library } \\ & \text { 2-3. } \text { Central Research Library } \\ & \text { 4. } \text { Reactor Division Library } \\ & \text { 5-6. Laboratory Records Dept. (LRD) } \\ & \text { 7. Laboratory Records (ORNL-RC) } \\ & \text { 8. ORNL Patent Office } \\ & \text { 9. ORNL Y-12 Technical Library } \\ & \text { 10. Socument Reference Section } \\ & \text { 11. C. E. Allman } \\ & \text { 12. R. D. Birkhoff } \\ & \text { 13. N. E. Brashier } \\ & \text { 14. T. A. Carlson } \\ & \text { 15. C. H. Chen } \\ & \text { 16. L. G. Christophorou }\end{aligned}$

17. R. N. Compton

18. W. R. Garrett

19-28. G. S. Hurst

29. J. P. Judish

30. S. V. Kaye

31. C. E. Klots

32. S. D. Kramer

33. M. G. Payne

34. H. Postma

35. C. R. Richmond

36. R. H. Ritchie

37. H. C. Schweinler

38. J. A. Stockdale

39. J. E. Turner

40. J. P. Young

EXTERNAL DISTRIBUTION

41. G. F. Bing, Lawrence Livermore Laboratory, P. 0. Box 808, Livermore, California 94550

42. J. E. Boring, Research Laboratory of Engineering Science, University of Virginia, University of Virginia, Charlottesville, Virginia 22901

43. K. Boyer, Division of Applied Photochemistry, LASL, Los Alamos, N.M. 87545

44. W. Brandt, Physics Department, New York University, New York, N.Y. 10003

45. R. S. Caswell, Radiation Physics Laboratory, NBS, Washington, D.C. 20234

46. L. W. Cochran, Vice President, University of Kentucky, Lexington, Kentuck.y 40506

47. G. Cowper, Radiation Dosimetry Branch, Atomic Energy of Canada Ltd., Chalk River, Ontario, Canada

48. W. C. DeMarcus, Department of Physics and Astronomy, University of Kentucky, Lexington, Kentucky 40506

49. J. Dubrin, Lawrence Livermore Laboratory, P. O. Box 808, Livermore, California 94550

50. F. Gabbard, Department of Physics and Astronomy, University of Kentucky, Lexington, Kentucky 40506

51. B. R. Gossick, Department of Physics and Astronomy, University of Kentucky, Lexington, Kentucky 40506

52. Alex E. S. Green, Department of Physics and Astronomy, University of Florida, Gainesville, Florida 32601

53. J. Grossman, College Station, Berea, Kentucky 40403 
54-58. L. W. Grossman, 6523 Ridge Circle, Cincinnati, Ohio 45213

59. T. W. Hansch, Department of Physics, Stanford University, Stanford, California 94305

60. M. Inokuti, Radiation Physics Division, Argonne National Laboratory, 9700 S. Cass Ave., Argonne, Illinois 60439

61. O'Dean Judd, Division of Applied Photochemistry, LASL, P. 0. Box 1663, Los Alamos, N.M. 87545

62. R. Katz, Department of Physics, University of Nebraska, Lincoln, Nebraska 68508

63. R. E. Knight, Department of Physics and Astronomy, University of Kentucky, Lexington, Kentucky 40506

64. P. K. Leichner, Department of Physics and Astronomy, University of Kentucky, Lexington, Kentucky 40506

65. J. L. Livermar, Assistant Administrator for Environment and Safety, ERDA, Washington, D.C. 20545

66. D. C. Lorents, Stanford Research Institute, Menlo Park, Salifornia 94025

67. J. L. Magee, Department of Chemistry, Lawrence Berkeley Laboratory, Berkeley, Califórn1a 94720

68. M. H. Nayfeh, Department of Physics, Yale University, New Haven, Connecticut 06520

69. E. A. O'Hair, Division of Applied Photochemistry, LASL, P. 0. Box 1663, Los Alamos, N.M. 87545

70. J. E. Parks, Department of Physics and Astronomy, Western Kentucky University, Bowling Green, Kentucky 42101

71. J. C. Person, Radiation Physics Division, Argonne National Laboratory, y/üU S. Làs̄s Áv̄e., Argonne, I1l1nols 60439

72. A. V. Phelps, Joint Inst1tute for Laboratory Astrophysics, Üniversiey of Colorudu, Buuldur, Culusadu 80302

73. C. P. Robinson, Division of Applied Photochemistry, LASL, P. 0. Box 1663, Los Alamos, N.M. 87545

74. H. H. Rossi, Radiological Research Laboratory, Columbia University, 630 W. 168th St., New York, N.Y. 10032

75. W. C. Royster, Dean of the Graduate School, University of Kentucky, Lexington, Kentucky 40506

76. A. L. Schawlow, Department of Physics, Stanford University, Stanford, California 94305

77. R. Schrills, Department of Physics and Astronomy, University of Kentucky, Lexington, Kentucky 40506

78. Maxine Sheets, Division of Biomedical and Environmental Research, ERDA, Washington, D.C. 20545

79. V. J. Sodd, Director, Nuclear Medicine Laboratory, Cincinnati General Hospital, Cincinnati, Ohio 45267

80. R. W. Solarz, Lawrence Livermore Laboratory, P. 0. Box 808, Livermore, California 94550

81. R. F. Stebbings, Department of Space Physics, Box 1892, Rice University, Houston, Texas 77001

82. T. D. Strickler, Department of Physics, Berea College, Berea, Kentucky 40403

83. D. A. Vroom, Intelcom Rad Tech, P. O. Box 80817, San Diego, California 92138 
84. R. W. Wood, Division of Biomedical and Environmental Research, ERDA, Washington, D.C. 20545

85. M. E. Wrenn, New York University Medical Center, Tuxedo, N.Y. 10987

86. ORAU Library

87. Research and Technical Support Division, ORO

88-114. Technical Information Center (TIC) 